\title{
Measurement of the $\gamma \gamma^{*} \rightarrow \pi^{0}$ transition form factor
}

B. Aubert, ${ }^{1}$ Y. Karyotakis, ${ }^{1}$ J. P. Lees, ${ }^{1}$ V. Poireau, ${ }^{1}$ E. Prencipe,${ }^{1}$ X. Prudent,${ }^{1}$ V. Tisserand, ${ }^{1}$ J. Garra Tico, ${ }^{2}$ E. Grauges,${ }^{2}$ M. Martinelli, ${ }^{3 a, 3 b}$ A. Palano, ${ }^{3 a, 3 b}$ M. Pappagallo, ${ }^{3 a, 3 b}$ G. Eigen, ${ }^{4}$ B. Stugu, ${ }^{4}$ L. Sun, ${ }^{4}$ M. Battaglia, ${ }^{5}$ D. N. Brown, ${ }^{5}$ L. T. Kerth, ${ }^{5}$ Yu. G. Kolomensky, ${ }^{5}$ G. Lynch, ${ }^{5}$ I. L. Osipenkov, ${ }^{5}$ K. Tackmann, ${ }^{5}$ T. Tanabe, ${ }^{5}$ C. M. Hawkes, ${ }^{6}$ N. Soni, ${ }^{6}$ A. T. Watson, ${ }^{6}$ H. Koch, ${ }^{7}$ T. Schroeder, ${ }^{7}$ D. J. Asgeirsson, ${ }^{8}$ B. G. Fulsom, ${ }^{8}$ C. Hearty, ${ }^{8}$ T. S. Mattison, ${ }^{8}$ J. A. McKenna, ${ }^{8}$ M. Barrett, ${ }^{9}$ A. Khan, ${ }^{9}$ A. Randle-Conde, ${ }^{9}$ V.E. Blinov, ${ }^{10}$ A. D. Bukin,,${ }^{10} *$ A. R. Buzykaev, ${ }^{10}$ V. P. Druzhinin, ${ }^{10}$ V. B. Golubev, ${ }^{10}$ A. P. Onuchin, ${ }^{10}$ S. I. Serednyakov, ${ }^{10}$ Yu. I. Skovpen, ${ }^{10}$ E. P. Solodov, ${ }^{10}$ K. Yu. Todyshev,${ }^{10}$ M. Bondioli, ${ }^{11}$ S. Curry, ${ }_{11}^{1}$ I. Eschrich, ${ }^{11}$ D. Kirkby, ${ }^{11}$ A. J. Lankford, ${ }^{11}$ P. Lund,${ }^{11}$ M. Mandelkern, ${ }^{11}$ E. C. Martin, ${ }^{11}$ D. P. Stoker, ${ }^{11}$ H. Atmacan, ${ }^{12}$ J. W. Gary, ${ }^{12}$ F. Liu, ${ }^{12}$ O. Long, ${ }^{12}$ G. M. Vitug, ${ }^{12}$ Z. Yasin, ${ }^{12}$ L. Zhang, ${ }^{12}$ V. Sharma,${ }^{13}$ C. Campagnari, ${ }^{14}$ T. M. Hong, ${ }^{14}$ D. Kovalskyi, ${ }^{14}$ M. A. Mazur, ${ }^{14}$ J. D. Richman, ${ }^{14}$ T. W. Beck,${ }^{15}$ A. M. Eisner, ${ }^{15}$ C. A. Heusch, ${ }^{15}$

J. Kroseberg, ${ }^{15}$ W. S. Lockman, ${ }^{15}$ A. J. Martinez, ${ }^{15}$ T. Schalk, ${ }^{15}$ B. A. Schumm, ${ }^{15}$ A. Seiden, ${ }^{15}$ L. Wang, ${ }^{15}$ L. O. Winstrom, ${ }^{15}$ C. H. Cheng, ${ }^{16}$ D. A. Doll, ${ }^{16}$ B. Echenard,${ }^{16}$ F. Fang, ${ }^{16}$ D. G. Hitlin, ${ }^{16}$ I. Narsky, ${ }^{16}$ T. Piatenko, ${ }^{16}$ F. C. Porter, ${ }^{16}$ R. Andreassen, ${ }^{17}$ G. Mancinelli, ${ }^{17}$ B. T. Meadows, ${ }^{17}$ K. Mishra, ${ }^{17}$ M. D. Sokoloff, ${ }^{17}$ P. C. Bloom, ${ }^{18}$ W. T. Ford, ${ }^{18}$ A. Gaz, ${ }^{18}$ J. F. Hirschauer, ${ }^{18}$ M. Nagel, ${ }^{18}$ U. Nauenberg, ${ }^{18}$ J. G. Smith, ${ }^{18}$ S. R. Wagner,${ }^{18}$ R. Ayad, ${ }^{19, \dagger}$ W. H. Toki, ${ }^{19}$ R. J. Wilson, ${ }^{19}$ E. Feltresi, ${ }^{20}$ A. Hauke, ${ }^{20}$ H. Jasper, ${ }^{20}$ T. M. Karbach, ${ }^{20}$ J. Merkel, ${ }^{20}$ A. Petzold, ${ }^{20}$ B. Spaan, ${ }^{20}$

K. Wacker, ${ }^{20}$ M. J. Kobel, ${ }^{21}$ R. Nogowski, ${ }^{21}$ K. R. Schubert, ${ }^{21}$ R. Schwierz, ${ }^{21}$ A. Volk, ${ }^{21}$ D. Bernard,${ }^{22}$ E. Latour, ${ }^{22}$

M. Verderi, ${ }^{22}$ P. J. Clark, ${ }^{23}$ S. Playfer, ${ }^{23}$ J. E. Watson, ${ }^{23}$ M. Andreotti, ${ }^{24 a, 24 b}$ D. Bettoni, ${ }^{24 a}$ C. Bozzi, ${ }^{24 a}$ R. Calabrese, ${ }^{24 a, 24 b}$ A. Cecchi, ${ }^{24 a, 24 b}$ G. Cibinetto, ${ }^{24 a, 24 b}$ E. Fioravanti, ${ }^{24 a, 24 b}$ P. Franchini, ${ }^{24 a, 24 b}$ E. Luppi, ${ }^{24 a, 24 b}$ M. Munerato, ${ }^{24 a, 24 b}$ M. Negrini, ${ }^{24 a, 24 b}$ A. Petrella, ${ }^{24 a, 24 b}$ L. Piemontese, ${ }^{24 a}$ V. Santoro, ${ }^{24 a, 24 b}$ R. Baldini-Ferroli, ${ }^{25}$ A. Calcaterra, ${ }^{25}$ R. de Sangro, ${ }^{25}$ G. Finocchiaro, ${ }^{25}$ S. Pacetti, ${ }^{25}$ P. Patteri, ${ }^{25}$ I. M. Peruzzi, ${ }^{25,}$ M. Piccolo, ${ }^{25}$ M. Rama, ${ }^{25}$ A. Zallo, ${ }^{25}$ R. Contri, ${ }^{26 a, 26 b}$ E. Guido, ${ }^{26 a, 26 b}$ M. Lo Vetere,${ }^{26 a, 26 b}$ M. R. Monge, ${ }^{26 a, 26 b}$ S. Passaggio, ${ }^{26 a}$ C. Patrignani, ${ }^{26 a, 26 b}$ E. Robutti, ${ }^{26 a}$ S. Tosi, ${ }^{26 a, 26 b}$ K. S. Chaisanguanthum, ${ }^{27}$ M. Morii, ${ }^{27}$ A. Adametz, ${ }^{28}$ J. Marks, ${ }^{28}$ S. Schenk,${ }^{28}$ U. Uwer, ${ }^{28}$ F. U. Bernlochner, ${ }^{29}$ V. Klose, ${ }^{29}$ H. M. Lacker, ${ }^{29}$ D. J. Bard, ${ }^{30}$ P. D. Dauncey, ${ }^{30}$ M. Tibbetts, ${ }^{30}$ P. K. Behera, ${ }^{31}$ M. J. Charles ${ }^{31}$ U. Mallik,${ }^{31}$ J. Cochran,${ }^{32}$ H. B. Crawley, ${ }^{32}$ L. Dong,${ }^{32}$ V. Eyges, ${ }^{32}$ W. T. Meyer, ${ }^{32}$ S. Prell, ${ }^{32}$ E. I. Rosenberg, ${ }^{32}$ A. E. Rubin, ${ }^{32}$ Y. Y. Gao, ${ }^{33}$ A. V. Gritsan, ${ }^{33}$ Z. J. Guo, ${ }^{33}$ N. Arnaud, ${ }^{34}$ J. Béquilleux,${ }^{34}$ A. D'Orazio, ${ }^{34}$ M. Davier, ${ }^{34}$ D. Derkach, ${ }^{34}$ J. Firmino da Costa, ${ }^{34}$ G. Grosdidier, ${ }^{34}$ F. Le Diberder, ${ }^{34}$ V. Lepeltier, ${ }^{34}$ A. M. Lutz, ${ }^{34}$ B. Malaescu, ${ }^{34}$ S. Pruvot, ${ }^{34}$ P. Roudeau,${ }^{34}$ M. H. Schune, ${ }^{34}$ J. Serrano, ${ }^{34}$ V. Sordini, ${ }^{34,8}$ A. Stocchi, ${ }^{34}$ G. Wormser, ${ }^{34}$ D. J. Lange, ${ }^{35}$ D. M. Wright, ${ }^{35}$ I. Bingham, ${ }^{36}$ J. P. Burke, ${ }^{36}$ C. A. Chavez, ${ }^{36}$ J. R. Fry, ${ }^{36}$ E. Gabathuler, ${ }^{36}$ R. Gamet, ${ }^{36}$

D. E. Hutchcroft, ${ }^{36}$ D. J. Payne, ${ }^{36}$ C. Touramanis, ${ }^{36}$ A. J. Bevan, ${ }^{37}$ C. K. Clarke, ${ }^{37}$ F. Di Lodovico, ${ }^{37}$ R. Sacco, ${ }^{37}$

M. Sigamani, ${ }^{37}$ G. Cowan, ${ }^{38}$ S. Paramesvaran, ${ }^{38}$ A. C. Wren, ${ }^{38}$ D. N. Brown, ${ }^{39}$ C. L. Davis, ${ }^{39}$ A. G. Denig, ${ }^{40}$ M. Fritsch, ${ }^{40}$ W. Gradl, ${ }^{40}$ A. Hafner, ${ }^{40}$ K. E. Alwyn, ${ }^{41}$ D. Bailey, ${ }^{41}$ R. J. Barlow, ${ }^{41}$ G. Jackson, ${ }^{41}$ G. D. Lafferty, ${ }^{41}$ T. J. West ${ }^{41}$ J. I. Yi ${ }^{41}$ J. Anderson, ${ }^{42}$ C. Chen, ${ }^{42}$ A. Jawahery, ${ }^{42}$ D. A. Roberts, ${ }^{42}$ G. Simi,${ }^{42}$ J. M. Tuggle,${ }^{42}$ C. Dallapiccola, ${ }^{43}$ E. Salvati, ${ }^{43}$ S. Saremi,${ }^{43}$ R. Cowan, ${ }^{44}$ D. Dujmic,${ }^{44}$ P. H. Fisher ${ }^{44}$ S. W. Henderson,,${ }^{44}$ G. Sciolla,${ }^{44}$ M. Spitznagel, ${ }^{44}$ R. K. Yamamoto, ${ }^{44}$ M. Zhao ${ }^{44}$ P. M. Patel, ${ }^{45}$ S. H. Robertson, ${ }^{45}$ M. Schram, ${ }^{45}$ A. Lazzaro, ${ }^{46 a, 46 b}$ V. Lombardo, ${ }^{46 a}$ F. Palombo, ${ }^{46 a, 46 b}$ S. Stracka, ${ }^{46 a, 46 b}$ J. M. Bauer, ${ }^{47}$ L. Cremaldi, ${ }^{47}$ R. Godang, ${ }^{47, \|}$ R. Kroeger, ${ }^{47}$ P. Sonnek, ${ }^{47}$ D. J. Summers, ${ }^{47}$ H. W. Zhao, ${ }^{47}$

M. Simard, ${ }^{48}$ P. Taras, ${ }^{48}$ H. Nicholson, ${ }^{49}$ G. De Nardo, ${ }^{50 a, 50 b}$ L. Lista, ${ }^{50 \mathrm{a}}$ D. Monorchio, ${ }^{50 \mathrm{a}, 50 \mathrm{~b}}$ G. Onorato, ${ }^{50 \mathrm{a}, 50 \mathrm{~b}}$

C. Sciacca, ${ }^{50 a, 50 b}$ G. Raven,${ }^{51}$ H. L. Snoek, ${ }^{51}$ C. P. Jessop, ${ }^{52}$ K. J. Knoepfel,${ }^{52}$ J. M. LoSecco, ${ }^{52}$ W. F. Wang, ${ }^{52}$ L. A. Corwin, ${ }^{53}$ K. Honscheid,${ }^{53}$ H. Kagan, ${ }^{53}$ R. Kass, ${ }^{53}$ J. P. Morris,${ }^{53}$ A. M. Rahimi,${ }^{53}$ J. J. Regensburger, ${ }^{53}$ S. J. Sekula, ${ }^{53}$ Q. K. Wong, ${ }^{53}$ N. L. Blount, ${ }^{54}$ J. Brau, ${ }^{54}$ R. Frey, ${ }^{54}$ O. Igonkina, ${ }^{54}$ J. A. Kolb,${ }^{54}$ M. Lu, ${ }^{54}$ R. Rahmat, ${ }^{54}$ N. B. Sinev, ${ }^{54}$

D. Strom,${ }^{54}$ J. Strube,${ }^{54}$ E. Torrence, ${ }^{54}$ G. Castelli, ${ }^{55,55 b}$ N. Gagliardi, ${ }^{55 a, 55 b}$ M. Margoni, ${ }^{55 a, 55 b}$ M. Morandin, ${ }^{55 a}$ M. Posocco, ${ }^{55 a}$ M. Rotondo, ${ }^{55 a}$ F. Simonetto,${ }^{55 a, 55 b}$ R. Stroili, ${ }^{55 a, 55 b}$ C. Voci, ${ }^{55 a, 55 b}$ P. del Amo Sanchez,${ }^{56}$ E. Ben-Haim, ${ }^{56}$ G. R. Bonneaud, ${ }^{56}$ H. Briand, ${ }^{56} \mathrm{~J}$. Chauveau, ${ }^{56} \mathrm{O}$. Hamon, ${ }^{56} \mathrm{Ph}$. Leruste, ${ }^{56} \mathrm{G}$. Marchiori, ${ }^{56} \mathrm{~J}$. Ocariz,${ }^{56} \mathrm{~A}$. Perez ${ }^{56}$

J. Prendki, ${ }^{56}$ S. Sitt, ${ }^{56}$ L. Gladney, ${ }^{57}$ M. Biasini, ${ }^{58 a, 58 b}$ E. Manoni, ${ }^{58 a, 58 b}$ C. Angelini, ${ }^{59 a, 59 b}$ G. Batignani, ${ }^{59 a, 59 b}$

S. Bettarini, ${ }^{59 a, 59 b}$ G. Calderini, ${ }^{59 a, 59 b, 4 l}$ M. Carpinelli, ${ }^{59 a, 59 b, * *}$ A. Cervelli, ${ }^{59 a, 59 b}$ F. Forti, ${ }^{59 a, 59 b}$ M. A. Giorgi, ${ }^{59 a, 59 b}$ A. Lusiani, ${ }^{59 a, 59 c}$ M. Morganti, ${ }^{59 a, 59 b}$ N. Neri, ${ }^{59 a, 59 b}$ E. Paoloni, ${ }^{59 a, 59 b}$ G. Rizzo, ${ }^{59 a, 59 b}$ J. J. Walsh, ${ }^{59 a}$ D. Lopes Pegna, ${ }^{\text {a60 }}$ C. Lu, ${ }^{a 60}$ J. Olsen, ${ }^{a 60}$ A. J. S. Smith, ${ }^{a 60}$ A. V. Telnov, ${ }^{a 60}$ F. Anulli, ${ }^{61 \mathrm{a}}$ E. Baracchini, ${ }^{61 \mathrm{a}, 61 \mathrm{~b}}$ G. Cavoto, ${ }^{61 \mathrm{a}}$ R. Faccini, ${ }^{61 \mathrm{a}, 61 \mathrm{~b}}$ F. Ferrarotto, ${ }^{61 \mathrm{a}}$ F. Ferroni, ${ }^{61 \mathrm{a}, 61 \mathrm{~b}}$ M. Gaspero, ${ }^{61 \mathrm{a}, 61 \mathrm{~b}}$ P. D. Jackson, ${ }^{61 \mathrm{a}} \mathrm{L}$. Li Gioi, ${ }^{61 \mathrm{a}}$ M. A. Mazzoni, ${ }^{61 \mathrm{a}}$ S. Morganti, ${ }^{61 \mathrm{a}}$ G. Piredda, ${ }^{61 \mathrm{a}}$ F. Renga, ${ }^{61 \mathrm{a}, 61 \mathrm{~b}}$ C. Voena ${ }^{61 \mathrm{a}}$ M. Ebert, ${ }^{62}$ T. Hartmann, ${ }^{62}$ H. Schröder, ${ }^{62}$ R. Waldi, ${ }^{62}$ T. Adye, ${ }^{63}$ B. Franek, ${ }^{63}$ E. O. Olaiya ${ }^{63}$ F. F. Wilson, ${ }^{63}$ S. Emery ${ }^{64}$ L. Esteve, ${ }^{64}$ G. Hamel de Monchenault, ${ }^{64}$ W. Kozanecki, ${ }^{64}$ G. Vasseur, ${ }^{64}$ Ch. Yèche ${ }^{64}$ M. Zito, ${ }^{64}$ M. T. Allen, ${ }^{65}$ D. Aston, ${ }^{65}$ R. Bartoldus, ${ }^{65}$ J. F. Benitez, ${ }^{65}$ R. Cenci, ${ }^{65}$ J. P. Coleman, ${ }^{65}$ 
M. R. Convery, ${ }^{65}$ J. C. Dingfelder, ${ }^{65}$ J. Dorfan, ${ }^{65}$ G. P. Dubois-Felsmann, ${ }^{65}$ W. Dunwoodie,${ }^{65}$ R. C. Field, ${ }^{65}$ M. Franco Sevilla, ${ }^{65}$ A. M. Gabareen, ${ }^{65}$ M. T. Graham, ${ }^{65}$ P. Grenier ${ }^{65}$ C. Hast,${ }^{65}$ W. R. Innes, ${ }^{65}$ J. Kaminski, ${ }^{65}$ M. H. Kelsey, ${ }^{65}$ H. Kim, ${ }^{65}$ P. Kim,${ }^{65}$ M. L. Kocian, ${ }^{65}$ D. W. G. S. Leith, ${ }^{65}$ S. Li ${ }^{65}$ B. Lindquist, ${ }^{65}$ S. Luitz, ${ }^{65}$ V. Luth, ${ }^{65}$ H. L. Lynch, ${ }^{65}$ D. B. MacFarlane, ${ }^{65}$ H. Marsiske, ${ }^{65}$ R. Messner, ${ }^{65,}$ ' D. R. Muller ${ }^{65}$ H. Neal,${ }^{65}$ S. Nelson, ${ }^{65}$ C. P. O' Grady, ${ }^{65}$ I. Ofte, ${ }^{65}$ M. Perl, ${ }^{65}$ B. N. Ratcliff, ${ }^{65}$ A. Roodman, ${ }^{65}$ A. A. Salnikov, ${ }^{65}$ R. H. Schindler, ${ }^{65}$ J. Schwiening, ${ }^{65}$ A. Snyder, ${ }^{65}$ D. Su, ${ }^{65}$ M. K. Sullivan, ${ }^{65}$ K. Suzuki, ${ }^{65}$ S. K. Swain, ${ }^{65}$ J. M. Thompson, ${ }^{65}$ J. Va'vra, ${ }^{65}$ A. P. Wagner,${ }^{65}$ M. Weaver ${ }^{65}$ C. A. West, ${ }^{65}$ W. J. Wisniewski, ${ }^{65}$ M. Wittgen,${ }^{65}$ D. H. Wright, ${ }^{65}$ H. W. Wulsin, ${ }^{65}$ A. K. Yarritu ${ }^{65}$ C. C. Young, ${ }^{65}$ V. Ziegler, ${ }^{65}$ X. R. Chen,${ }^{66}$ H. Liu, ${ }^{66}$ W. Park, ${ }^{66}$ M. V. Purohit, ${ }^{66}$ R. M. White, ${ }^{66}$ J. R. Wilson, ${ }^{66}$ P. R. Burchat, ${ }^{67}$ A. J. Edwards, ${ }^{67}$ T. S. Miyashita, ${ }^{67}$ S. Ahmed, ${ }^{68}$ M. S. Alam, ${ }^{68}$ J. A. Ernst, ${ }^{68}$ B. Pan,${ }^{68}$ M. A. Saeed, ${ }^{68}$ S. B. Zain, ${ }^{68}$ A. Soffer, ${ }^{69}$ S. M. Spanier, ${ }^{70}$ B. J. Wogsland, ${ }^{70}$ R. Eckmann, ${ }^{71}$ J. L. Ritchie, ${ }^{71}$ A. M. Ruland, ${ }^{71}$ C. J. Schilling, ${ }^{71}$ R. F. Schwitters, ${ }^{71}$ B. C. Wray, ${ }^{71}$ B. W. Drummond, ${ }^{72}$ J. M. Izen, ${ }^{72}$ X. C. Lou, ${ }^{72}$ F. Bianchi, ${ }^{73 a, 73 b}$ D. Gamba, ${ }^{73 a, 73 b}$ M. Pelliccioni ${ }^{73 a, 73 b}$ M. Bomben, ${ }^{74 a, 74 b}$ L. Bosisio, ${ }^{74 a, 74 b}$ C. Cartaro, ${ }^{74 a, 74 b}$ G. Della Ricca, ${ }^{74 a, 74 b}$ L. Lanceri, ${ }^{74 a, 74 b}$ L. Vitale,${ }^{74 a, 74 b}$ V. Azzolini, ${ }^{75}$ N. Lopez-March, ${ }^{75}$ F. Martinez-Vidal,${ }^{75}$ D. A. Milanes, ${ }^{75}$ A. Oyanguren, ${ }^{75}$ J. Albert, ${ }^{76}$ Sw. Banerjee, ${ }^{76}$ B. Bhuyan,${ }^{76}$ H. H. F. Choi,${ }^{76}$ K. Hamano, ${ }^{76}$ G. J. King ${ }^{76}$ R. Kowalewski, ${ }^{76}$ M. J. Lewczuk, ${ }^{76}$ I. M. Nugent, ${ }^{76}$ J. M. Roney, ${ }^{76}$ R. J. Sobie, ${ }^{76}$ T. J. Gershon, ${ }^{77}$ P. F. Harrison, ${ }^{77}$ J. Ilic, ${ }^{77}$ T. E. Latham, ${ }^{77}$ G. B. Mohanty, ${ }^{77}$ E. M. T. Puccio, ${ }^{77}$ H. R. Band,${ }^{78}$ X. Chen,${ }^{78}$ S. Dasu, ${ }^{78}$ K. T. Flood, ${ }^{78}$ Y. Pan,${ }^{78}$ R. Prepost,${ }^{78}$ C. O. Vuosalo, ${ }^{78}$ and S. L. $\mathrm{Wu}^{78}$

(BABAR Collaboration)

\author{
${ }^{1}$ Laboratoire d'Annecy-le-Vieux de Physique des Particules (LAPP), Université de Savoie, \\ CNRS/IN2P3, F-74941 Annecy-Le-Vieux, France \\ ${ }^{2}$ Facultat de Fisica, Departament ECM, Universitat de Barcelona, E-08028 Barcelona, Spain \\ ${ }^{3 a}$ INFN Sezione di Bari, I-70126 Bari, Italy \\ ${ }^{3 \mathrm{~b}}$ Dipartimento di Fisica, Università di Bari, I-70126 Bari, Italy \\ ${ }^{4}$ Institute of Physics, University of Bergen, N-5007 Bergen, Norway \\ ${ }^{5}$ Lawrence Berkeley National Laboratory and University of California, Berkeley, California 94720, USA \\ ${ }^{6}$ University of Birmingham, Birmingham, B15 2TT, United Kingdom \\ ${ }^{7}$ Institut für Experimentalphysik 1, Ruhr Universität Bochum, D-44780 Bochum, Germany \\ ${ }^{8}$ University of British Columbia, Vancouver, British Columbia, Canada V6T IZI \\ ${ }^{9}$ Brunel University, Uxbridge, Middlesex UB8 3PH, United Kingdom \\ ${ }^{10}$ Budker Institute of Nuclear Physics, Novosibirsk 630090, Russia \\ ${ }^{11}$ University of California at Irvine, Irvine, California 92697, USA \\ ${ }^{12}$ University of California at Riverside, Riverside, California 92521, USA \\ ${ }^{13}$ University of California at San Diego, La Jolla, California 92093, USA \\ ${ }^{14}$ University of California at Santa Barbara, Santa Barbara, California 93106, USA \\ ${ }^{15}$ Institute for Particle Physics, University of California at Santa Cruz, Santa Cruz, California 95064, USA \\ ${ }^{16}$ California Institute of Technology, Pasadena, California 91125, USA \\ ${ }^{17}$ University of Cincinnati, Cincinnati, Ohio 45221, USA \\ ${ }^{18}$ University of Colorado, Boulder, Colorado 80309, USA \\ ${ }^{19}$ Colorado State University, Fort Collins, Colorado 80523, USA \\ ${ }^{20}$ Technische Universität Dortmund, Fakultät Physik, D-44221 Dortmund, Germany \\ ${ }^{21}$ Institut für Kern-und Teilchenphysik, Technische Universität Dresden, D-01062 Dresden, Germany \\ ${ }^{22}$ Laboratoire Leprince-Ringuet, CNRS/IN2P3, Ecole Polytechnique, F-91128 Palaiseau, France \\ ${ }^{23}$ University of Edinburgh, Edinburgh EH9 3JZ, United Kingdom \\ ${ }^{24 a}$ INFN Sezione di Ferrara, I-44100 Ferrara, Italy \\ ${ }^{24 \mathrm{~b}}$ Dipartimento di Fisica, Università di Ferrara, I-44100 Ferrara, Italy \\ ${ }^{25}$ INFN Laboratori Nazionali di Frascati, I-00044 Frascati, Italy \\ ${ }^{26 a}$ INFN Sezione di Genova, I-16146 Genova, Italy \\ ${ }^{26 \mathrm{~b}}$ Dipartimento di Fisica, Università di Genova, I-16146 Genova, Italy \\ ${ }^{27}$ Harvard University, Cambridge, Massachusetts 02138, USA \\ ${ }^{28}$ Physikalisches Institut, Universität Heidelberg, Philosophenweg 12, D-69120 Heidelberg, Germany \\ ${ }^{29}$ Institut für Physik, Humboldt-Universität zu Berlin, Newtonstrasse 15, D-12489 Berlin, Germany \\ ${ }^{30}$ Imperial College London, London, SW7 2AZ, United Kingdom \\ ${ }^{31}$ University of Iowa, Iowa City, Iowa 52242, USA \\ ${ }^{32}$ Iowa State University, Ames, Iowa 50011-3160, USA \\ ${ }^{33}$ Johns Hopkins University, Baltimore, Maryland 21218, USA
}


${ }^{34}$ Laboratoire de l'Accélérateur Linéaire, IN2P3/CNRS et Université Paris-Sud 11,

Centre Scientifique d'Orsay, B.P. 34, F-91898 Orsay Cedex, France

${ }^{35}$ Lawrence Livermore National Laboratory, Livermore, California 94550, USA

${ }^{36}$ University of Liverpool, Liverpool L69 7ZE, United Kingdom

${ }^{37}$ Queen Mary, University of London, London, E1 4NS, United Kingdom

${ }^{38}$ University of London, Royal Holloway and Bedford New College, Egham, Surrey TW20 OEX, United Kingdom

${ }^{39}$ University of Louisville, Louisville, Kentucky 40292, USA

${ }^{40}$ Institut für Kernphysik, Johannes Gutenberg-Universität Mainz, D-55099 Mainz, Germany

${ }^{41}$ University of Manchester, Manchester M13 9PL, United Kingdom

${ }^{42}$ University of Maryland, College Park, Maryland 20742, USA

${ }^{43}$ University of Massachusetts, Amherst, Massachusetts 01003, USA

${ }^{44}$ Laboratory for Nuclear Science, Massachusetts Institute of Technology, Cambridge, Massachusetts 02139, USA

${ }^{45}$ McGill University, Montréal, Québec, Canada H3A 2 T8

${ }^{46 a}$ INFN Sezione di Milano, I-20133 Milano, Italy

${ }^{46 \mathrm{~b}}$ Dipartimento di Fisica, Università di Milano, I-20133 Milano, Italy

${ }^{47}$ University of Mississippi, University, Mississippi 38677, USA

${ }^{48}$ Physique des Particules, Université de Montréal, Montréal, Québec, Canada H3C 3J7

${ }^{49}$ Mount Holyoke College, South Hadley, Massachusetts 01075, USA

${ }^{50 a}$ INFN Sezione di Napoli, I-80126 Napoli, Italy

${ }^{50 \mathrm{~b}}$ Dipartimento di Scienze Fisiche, Università di Napoli Federico II, I-80126 Napoli, Italy

${ }^{51}$ NIKHEF, National Institute for Nuclear Physics and High Energy Physics, NL-1009 DB Amsterdam, The Netherlands

${ }^{52}$ University of Notre Dame, Notre Dame, Indiana 46556, USA

${ }^{53}$ Ohio State University, Columbus, Ohio 43210, USA

${ }^{54}$ University of Oregon, Eugene, Oregon 97403, USA

${ }^{55 a}$ INFN Sezione di Padova, I-35131 Padova, Italy

${ }^{55 b}$ Dipartimento di Fisica, Università di Padova, I-35131 Padova, Italy

${ }^{56}$ Laboratoire de Physique Nucléaire et de Hautes Energies, IN2P3/CNRS, Université Pierre et Marie Curie-Paris6, Université Denis Diderot-Paris7, F-75252 Paris, France

${ }^{57}$ University of Pennsylvania, Philadelphia, Pennsylvania 19104, USA

${ }^{58}$ INFN Sezione di Perugia, I-06100 Perugia, Italy

${ }^{58 \mathrm{~b}}$ Dipartimento di Fisica, Università di Perugia, I-06100 Perugia, Italy

${ }^{59 a}$ INFN Sezione di Pisa, I-56127 Pisa, Italy

${ }^{59 \mathrm{~b}}$ Dipartimento di Fisica, Università di Pisa, I-56127 Pisa, Italy

${ }^{59 \mathrm{c}}$ Scuola Normale Superiore di Pisa, I-56127 Pisa, Italy

${ }^{\mathrm{a} 60}$ Princeton University, Princeton, New Jersey 08544, USA

${ }^{61}$ INFN Sezione di Roma, I-00185 Roma, Italy

${ }^{61 b}$ Dipartimento di Fisica, Università di Roma La Sapienza, I-00185 Roma, Italy

${ }^{62}$ Universität Rostock, D-18051 Rostock, Germany

${ }^{63}$ Rutherford Appleton Laboratory, Chilton, Didcot, Oxon, OX11 OQX, United Kingdom

${ }^{64}$ CEA, Irfu, SPP, Centre de Saclay, F-91191 Gif-sur-Yvette, France

${ }^{65}$ SLAC National Accelerator Laboratory, Stanford, California 94309 USA

${ }^{66}$ University of South Carolina, Columbia, South Carolina 29208, USA

${ }^{67}$ Stanford University, Stanford, California 94305-4060, USA

${ }^{68}$ State University of New York, Albany, New York 12222, USA

${ }^{69}$ School of Physics and Astronomy, Tel Aviv University, Tel Aviv, 69978, Israel

${ }^{70}$ University of Tennessee, Knoxville, Tennessee 37996, USA

${ }^{71}$ University of Texas at Austin, Austin, Texas 78712, USA

${ }^{72}$ University of Texas at Dallas, Richardson, Texas 75083, USA

${ }^{73 a}$ INFN Sezione di Torino, I-10125 Torino, Italy

${ }^{73 b}$ Dipartimento di Fisica Sperimentale, Università di Torino, I-10125 Torino, Italy

${ }^{74 a}$ INFN Sezione di Trieste, I-34127 Trieste, Italy

${ }^{74 \mathrm{~b}}$ Dipartimento di Fisica, Università di Trieste, I-34127 Trieste, Italy

${ }^{75}$ IFIC, Universitat de Valencia-CSIC, E-46071 Valencia, Spain

\footnotetext{
*Deceased.

${ }^{\dagger}$ Now at Temple University, Philadelphia, Pennsylvania 19122, USA.

‡Also with Università di Perugia, Dipartimento di Fisica, Perugia, Italy.

${ }^{\S}$ Also with Università di Roma La Sapienza, I-00185 Roma, Italy.
}

\footnotetext{
"Now at University of South Alabama, Mobile, Alabama 36688, USA.

${ }^{\text {II }}$ Also with Laboratoire de Physique Nucléaire et de Hautes Energies, IN2P3/CNRS, Université Pierre et Marie Curie-Paris 6, Université Denis Diderot-Paris 7, F-75252 Paris, France.

**Also with Università di Sassari, Sassari, Italy.
} 


\author{
${ }^{76}$ University of Victoria, Victoria, British Columbia, Canada V8W $3 P 6$ \\ ${ }^{77}$ Department of Physics, University of Warwick, Coventry CV4 7AL, United Kingdom \\ ${ }^{78}$ University of Wisconsin, Madison, Wisconsin 53706, USA
}

(Received 29 May 2009; published 11 September 2009)

\begin{abstract}
We study the reaction $e^{+} e^{-} \rightarrow e^{+} e^{-} \pi^{0}$ in the single tag mode and measure the differential cross section $d \sigma / d Q^{2}$ and the $\gamma \gamma^{*} \rightarrow \pi^{0}$ transition form factor in the momentum transfer range from 4 to $40 \mathrm{GeV}^{2}$. At $Q^{2}>10 \mathrm{GeV}^{2}$ the measured form factor exceeds the asymptotic limit predicted by perturbative QCD. The analysis is based on $442 \mathrm{fb}^{-1}$ of integrated luminosity collected at PEP-II with the $B A B A R$ detector at $e^{+} e^{-}$center-of-mass energies near $10.6 \mathrm{GeV}$.
\end{abstract}

DOI: 10.1103/PhysRevD.80.052002

PACS numbers: 14.40.Aq, 13.40.Gp, 12.38.Qk

\section{INTRODUCTION}

In this paper we study the process

$$
e^{+} e^{-} \rightarrow e^{+} e^{-} \pi^{0},
$$

where the final state $\pi^{0}$ is produced via the two-photon production mechanism illustrated by Fig. 1. We measure the differential cross section for this process in the single tag mode where one of the outgoing electrons ${ }^{1}$ (tagged) is detected while the other electron (untagged) is scattered at a small angle. The $\pi^{0}$ is observed through its decay into two photons. The tagged electron emits a highly off-shell photon with the momentum transfer $q_{1}^{2} \equiv-Q^{2}=$ $\left(p-p^{\prime}\right)^{2}$, where $p$ and $p^{\prime}$ are the four momenta of the initial and final electrons. The momentum transfer to the untagged electron is near zero. The differential cross section for pseudoscalar meson production $d \sigma\left(e^{+} e^{-} \rightarrow\right.$ $\left.e^{+} e^{-} \pi^{0}\right) / d Q^{2}$ depends on only one form factor, $F\left(Q^{2}\right)$, which describes the $\gamma \gamma^{*} \rightarrow \pi^{0}$ transition. To relate the differential cross section to the transition form factor we use the formulas for the $e^{+} e^{-} \rightarrow e^{+} e^{-} \pi^{0}$ cross section in Eqs. (2.1) and (4.5) of Ref. [1].

At large momentum transfer, $Q^{2}$, perturbative QCD (pQCD) predicts that the transition form factor can be represented as a convolution of a calculable hard-scattering amplitude for $\gamma \gamma^{*} \rightarrow q \bar{q}$ with a nonperturbative pion distribution amplitude, $\phi_{\pi}\left(x, Q^{2}\right)$ [2]. The latter can be interpreted as the amplitude for the transition of the pion with momentum $P$ into two quarks with momenta $P x$ and $P(1-$ $x$ ). In lowest order $\mathrm{pQCD}$ the transition form factor is obtained from

$$
\begin{aligned}
Q^{2} F\left(Q^{2}\right)= & \frac{\sqrt{2} f_{\pi}}{3} \int_{0}^{1} \frac{d x}{x} \phi_{\pi}\left(x, Q^{2}\right)+O\left(\alpha_{s}\right) \\
& +O\left(\frac{\Lambda_{\mathrm{QCD}}^{2}}{Q^{2}}\right),
\end{aligned}
$$

where $f_{\pi}=0.131 \mathrm{GeV}$ is the pion decay constant. The pion distribution amplitude (DA) plays an important role in theoretical descriptions of many hard-scattering QCD processes. Since the evolution of $\phi_{\pi}\left(x, Q^{2}\right)$ with $Q^{2}$ is pre-

\footnotetext{
${ }^{1}$ Unless otherwise specified, we use the term "electron" for either an electron or a positron.
}

dicted by $\mathrm{pQCD}$, experimental data on the transition form factor can be used to determine its unknown dependence on $x$ [3-10].

The pion transition form factor was measured in the CELLO [11] and CLEO [12] experiments in the momentum transfer ranges $0.7-2.2 \mathrm{GeV}^{2}$ and $1.6-8.0 \mathrm{GeV}^{2}$, respectively. In this paper we study the form factor in the $Q^{2}$ range from 4 to $40 \mathrm{GeV}^{2}$.

\section{THE BABAR DETECTOR AND DATA SAMPLES}

We analyze a data sample corresponding to an integrated luminosity of about $442 \mathrm{fb}^{-1}$ recorded with the BABAR detector [13] at the PEP-II asymmetric-energy storage rings. At PEP-II, 9-GeV electrons collide with $3.1-\mathrm{GeV}$ positrons to yield a center-of-mass energy of $10.58 \mathrm{GeV}$ [the $Y(4 \mathrm{~S})$ resonance]. Additional data $\left(\sim 43 \mathrm{fb}^{-1}\right)$ recorded at $10.54 \mathrm{GeV}$ for the purpose of $Y(4 \mathrm{~S})$ background studies are included in the present analysis.

Charged-particle tracking is provided by a five-layer silicon vertex tracker (SVT) and a 40-layer drift chamber $(\mathrm{DCH})$, operating in a 1.5-T axial magnetic field. The transverse momentum resolution is $0.47 \%$ at $1 \mathrm{GeV} / c$. Energies of photons and electrons are measured with a CsI(Tl) electromagnetic calorimeter (EMC) with a resolution of $3 \%$ at $1 \mathrm{GeV}$. Charged-particle identification is provided by specific ionization $(d E / d x)$ measurements in the SVT and DCH, and by an internally reflecting ringimaging Cherenkov detector (DIRC).

Signal and background $e^{+} e^{-} \rightarrow e^{+} e^{-} \pi^{0} \pi^{0}$ processes are simulated with the Monte Carlo (MC) event generator GGRESRC. It uses the formula for the differential cross

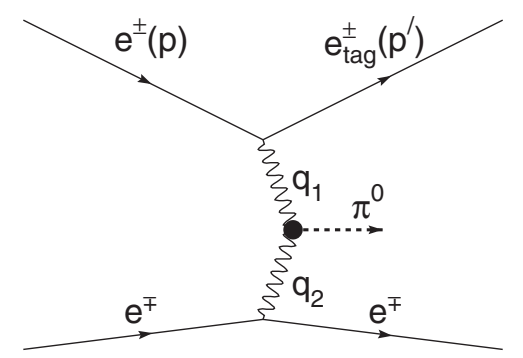

FIG. 1. The Feynman diagram for the $e^{+} e^{-} \rightarrow e^{+} e^{-} \pi^{0}$ twophoton production process. 
section from Ref. [1] for $\pi^{0}$ production and the BudnevGinzburg-Meledin-Serbo formalism [14] for the two pion final state. Because the $Q^{2}$ distribution is peaked near zero, the $\mathrm{MC}$ events are generated with a restriction on the momentum transfer to one of the electrons: $Q^{2}=-q_{1}^{2}>$ $3 \mathrm{GeV}^{2}$. This restriction corresponds to the limit of detector acceptance for the tagged electron. The second electron is required to have momentum transfer $-q_{2}^{2}<0.6 \mathrm{GeV}^{2}$. The experimental criterion providing this restriction for data events is described in Sec. III. The pseudoscalar form factor is fixed to $F(0)$ in MC simulation.

The GGRESRC event generator includes next-to-leadingorder radiative corrections to the Born cross section calculated according to Ref. [15]. In particular, it generates extra soft photons emitted by the initial and final state electrons. The formulas from Ref. [15] were modified to account for the hadron contribution to the vacuum polarization diagrams. The maximum energy of the extra photon emitted from the initial state is restricted by the requirement ${ }^{2} E_{\gamma}^{*}<$ $0.05 \sqrt{s}$, where $\sqrt{s}$ is the $e^{+} e^{-}$center-of-mass (c.m.) energy. The generated events are subjected to detailed detector simulation based on GEANT4 [16], and are reconstructed with the software chain used for the experimental data. Variations in the detector and beam background conditions are taken into account. In particular, we simulate the beam-induced background, which may lead to the appearance of extra photons and tracks in the events of interest, by overlaying the raw data from a random trigger event on each generated event.

Background events from $e^{+} e^{-} \rightarrow q \bar{q}$, where $q$ represents a $u, d, s$, or $c$ quark, $e^{+} e^{-} \rightarrow \tau^{+} \tau^{-}$, and $e^{+} e^{-} \rightarrow B \bar{B}$ are simulated with the JETSET [17], KK2F [18], and EVTGEN [19] event generators, respectively.

\section{EVENT SELECTION}

At the trigger level candidate events for the process under study are selected by the VirtualCompton filter. This filter was originally designed to select so-called virtual Compton scattering (VCS) events used for detector calibration. This process corresponds to $e^{+} e^{-} \rightarrow e^{+} e^{-} \gamma$ with the kinematic requirement that one of the final state electrons goes along the collision axis, while the other electron and the photon are scattered at large angles. The filter requires that a candidate event contain a track with $p^{*} / \sqrt{s}>0.1$ and a cluster in the EMC with $E^{*} / \sqrt{s}>0.1$ which is approximately opposite in azimuth $\left(\left|\delta \phi^{*}-\pi\right|<\right.$ $0.1 \mathrm{rad})$ to this track. Cluster and track polar angle acolinearity in the c.m. frame is required to be greater than $0.1 \mathrm{rad}$. Finally, the measured missing energy in the c.m. frame, which should correspond to the undetected electron, is compared to a prediction based entirely on the directions of the detected particles, and the assumption that the

\footnotetext{
${ }^{2}$ Throughout this paper the asterisk denotes quantities in the $e^{+} e^{-}$c.m. frame.
}

missing momentum is directed along the collision axis: $\left|E_{\text {meas }}^{*}-E_{\text {pred }}^{*}\right| / \sqrt{s}<0.05$. For a significant fraction of the $e^{+} e^{-} \rightarrow e^{+} e^{-} \pi^{0}$ events, the trigger cluster algorithm cannot separate the photons from $\pi^{0}$ decay, and hence identifies them as a single photon. Therefore the VirtualCompton filter has relatively large efficiency (about 50\%-80\% depending on the $\pi^{0}$ energy) for signal events.

In each event selected by the VirtualCompton filter, we search for an electron and a $\pi^{0}$ candidate. A charged track identified as an electron must originate from the interaction point and be in the polar angle range $0.376<\theta_{e}<$ $2.450 \mathrm{rad}$ in the laboratory frame. The latter requirement is needed to provide high efficiency for the trigger trackfinding algorithm and for good electron identification. To recover electron energy loss due to bremsstrahlung, both internal and in the detector material before the DCH, we look for EMC showers close to the electron direction and combine their energies with the measured energy of the electron track. The resulting laboratory energy of the electron candidate must be greater than $2 \mathrm{GeV}$. Two photon candidates with energies greater than $50 \mathrm{MeV}$ are combined to form a $\pi^{0}$ candidate by requiring that their invariant mass be in the range $0.06-0.21 \mathrm{GeV} / c^{2}$ and that their laboratory energy sum be greater than $1.5 \mathrm{GeV}$. Since a significant fraction of events contains beam-generated spurious track and photon candidates, extra tracks and extra photons are allowed in an event.

The main background process, VCS, has a cross section several thousand times greater than that for the process under study. The VCS photon together with a soft photon, for example from beam background, may give an invariant mass value close to the $\pi^{0}$ mass. Such background events are effectively rejected by requirements on the photon helicity angle $\left(\left|\cos \theta_{h}\right|<0.8\right)$ and on the $\pi^{0}$ c.m. polar angle $\left(\left|\cos \theta_{\pi}^{*}\right|<0.8\right)$. The photon helicity angle $\theta_{h}$ is defined as the angle between the decay photon momentum in the $\pi^{0}$ rest frame and the $\pi^{0}$ direction in the laboratory frame.

The next step is to remove improperly reconstructed QED events. We remove events which involve noisy EMC channels, events with extra tracks close to the $\pi^{0}$ candidate direction, and events with $\left|\Delta \theta_{\gamma \gamma}\right|<0.025 \mathrm{rad}$, where $\Delta \theta_{\gamma \gamma}$ is the difference between the laboratory polar angles of the photons from the $\pi^{0}$ decay. The latter condition removes VCS events where the photon converted to an $e^{+} e^{-}$pair within the $\mathrm{DCH}$ volume. It also removes about $20 \%$ of the signal events, but significantly improves (by a factor of about 15) the signal-to-background ratio.

Two additional event kinematics requirements provide further background suppression and improved data to MCsimulation correspondence. Figure 2 shows the data and MC-simulation distributions of the cosine of the polar angle of the momentum vector of the $e \pi^{0}$ system in the c.m. frame. We require $\left|\cos \theta_{e \pi}^{*}\right|>0.99$. This effectively limits the value of the momentum transfer to the untagged 


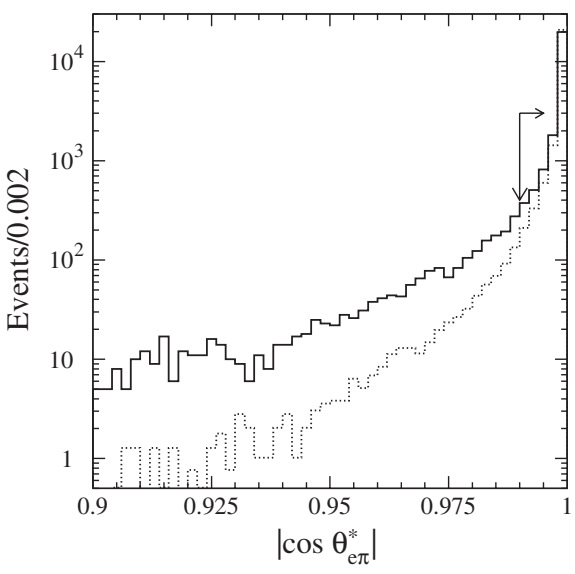

FIG. 2. The distribution of the cosine of the polar angle of the $e \pi^{0}$ system momentum in the c.m. frame for data (solid histogram) and simulated signal (dotted histogram). Events for which $\left|\cos \theta_{e \pi}^{*}\right|>0.99$ (indicated by the arrow) are retained.

electron $\left(q_{2}^{2}\right)$ and guarantees compliance with the condition $-q_{2}^{2}<0.6 \mathrm{GeV}^{2}$ used in $\mathrm{MC}$ simulation.

The emission of extra photons by the electrons involved leads to a difference between the measured and actual values of $Q^{2}$. In the case of initial state radiation (ISR) $Q_{\text {meas }}^{2}=Q_{\text {true }}^{2}\left(1+r_{\gamma}\right)$, where $r_{\gamma}=2 E_{\gamma}^{*} / \sqrt{s}$. To restrict the energy of the ISR photon we use the parameter

$$
r=\frac{\sqrt{s}-E_{e \pi}^{*}-p_{e \pi}^{*}}{\sqrt{s}}
$$

where $E_{e \pi}^{*}$ and $p_{e \pi}^{*}$ are the c.m. energy and the magnitude of the momentum of the detected $e \pi^{0}$ system. In the ISR case this parameter coincides with $r_{\gamma}$ defined above. The condition $r<0.075$ ensures compliance with the restriction $r_{\gamma}<0.1$ used in MC simulation. The $r$ distribution for data is shown in Fig. 3, where the shaded histogram shows

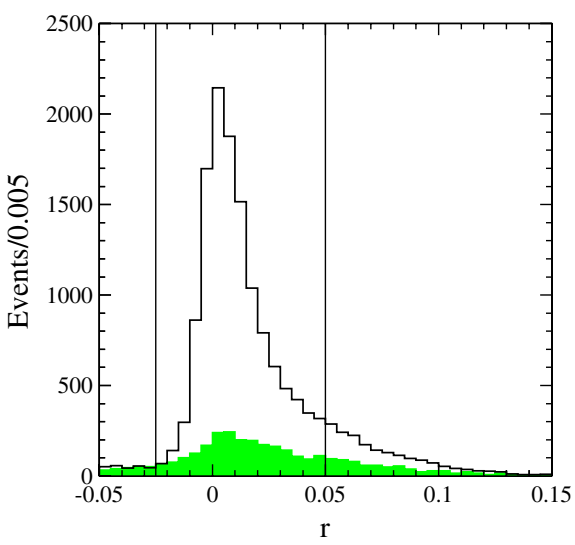

FIG. 3 (color online). The $r$ distribution for data events. The shaded histogram shows the background contribution estimated from the fit to the two-photon mass distribution (Sec. IV). The vertical lines indicate the region used to select candidate events $(-0.025<r<0.050)$.

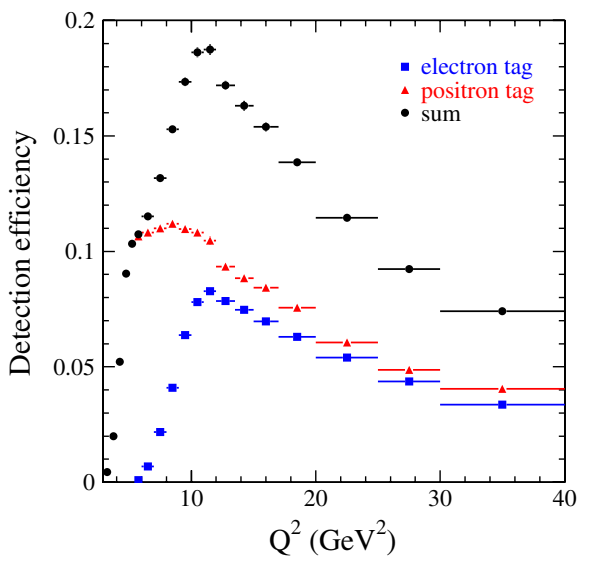

FIG. 4 (color online). The detection efficiency as a function of the momentum transfer squared for events with a tagged electron (squares), a tagged positron (triangles), and their sum (circles).

the background estimated from the fit to the two-photon mass distribution (Sec. IV). We select events with $-0.025<r<0.050$ for further analysis.

The background from $e^{+} e^{-}$annihilation into hadrons is strongly suppressed by the requirements of electron identification, on $\cos \theta_{e \pi}^{*}$, and on $r$. An additional twofold suppression of this background is provided by the condition that the $z$ component of the c.m. momentum of the $e \pi^{0}$ system is negative (positive) for events with a tagged positron (electron).

The $Q^{2}$ dependence of the detection efficiency obtained from MC simulation is shown in Fig. 4. The detector acceptance limits the detection efficiency at small $Q^{2}$. To avoid possible systematics due to data-simulation differences near detector edges, we measure the cross section and form factor in the region $Q^{2}>4 \mathrm{GeV}^{2}$. The asymmetry of the $e^{+} e^{-}$collisions at PEP-II leads to different efficiencies for events with electron and positron tags. The $Q^{2}$ range from 4 to $7 \mathrm{GeV}^{2}$ is measured only with the positron tag. The decrease of the detection efficiency in the region $Q^{2}>10 \mathrm{GeV}^{2}$ is explained by the decrease of the $\pi^{0}$ reconstruction efficiency due to growth of the average $\pi^{0}$ energy with $Q^{2}$.

The efficiency corrections and systematic uncertainties due to imperfect simulation of detector response are considered in Sec. VI.

\section{FITTING THE TWO-PHOTON MASS SPECTRUM}

The two-photon mass spectrum for selected data events with $4<Q^{2}<40 \mathrm{GeV}^{2}$ is shown in Fig. 5; for $Q^{2}>$ $40 \mathrm{GeV}^{2}$ we do not see evidence of a $\pi^{0}$ signal over background. To determine the number of events containing a $\pi^{0}$, we perform a binned likelihood fit to the spectrum with a sum of signal and background distributions. We describe the signal line shape by a sum of two $F_{B 1}$ functions with the same position of their maxima [20]. The 


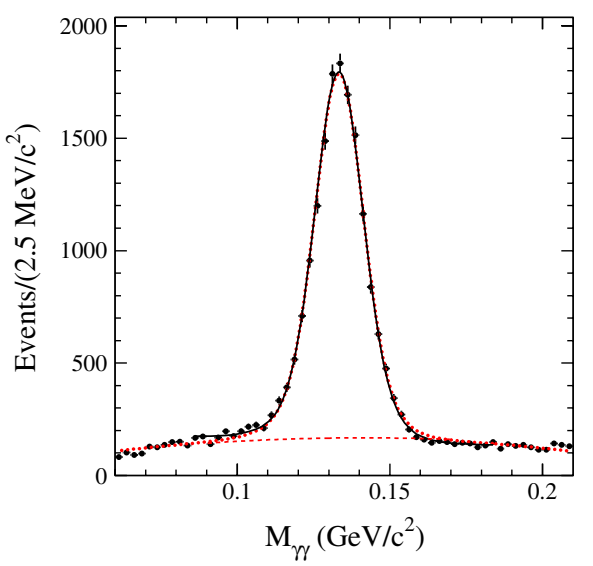

FIG. 5 (color online). The two-photon invariant mass spectrum for data events with $4<Q^{2}<40 \mathrm{GeV}^{2}$. The solid (dotted) curve corresponds to the fit with a linear (quadratic) background shape. The dashed curve represents the fitted quadratic background.

function $F_{B 1}$ is the convolution of a Gaussian and an exponential distribution:

$$
\begin{aligned}
F_{B 1}\left(x ; x_{g}, \sigma_{g}, \lambda\right)= & \frac{1}{2|\lambda|} \exp \left(-\frac{x-x_{g}}{\lambda}+\frac{\sigma_{g}^{2}}{2 \lambda^{2}}\right) \\
& \times\left[1-\operatorname{erf}\left(\frac{\sigma_{g}^{2}-\left(x-x_{g}\right) \lambda}{\sqrt{2} \sigma_{g}|\lambda|}\right)\right] .
\end{aligned}
$$

The parameters of the $\pi^{0}$ resolution function are fixed from the fit of the mass spectrum obtained for simulated signal events weighted to yield the $Q^{2}$ dependence observed in data. The background distribution is described either by a linear function in the mass range $0.085-0.185 \mathrm{GeV} / c^{2}$ or a second order polynomial in the mass range $0.06-0.21 \mathrm{GeV} / c^{2}$. The data mass spectrum is fitted with five (six for second order polynomial) free parameters: the number of signal events, the peak position, the sigma of one (narrow) of the $F_{B 1}$ functions $\left(\sigma_{1}\right)$, and two (three) parameters for the background. The results of the fits are shown in Fig. 5.

The total number of signal events is about 14000 . The difference in signal yield between the two background hypotheses is 170 events, while the statistical error on the signal yield is 140 events. The difference between the peak positions in data and MC simulation is consistent with zero. The value of $\sigma_{1}$ is $7.5 \mathrm{MeV} / c^{2}$ in data and 7.7 $\mathrm{MeV} / c^{2}$ in simulation, which corresponds to a difference of about two standard deviations.

A similar fitting procedure is applied in each of the seventeen $Q^{2}$ intervals indicated in Table I. The parameters of the $\pi^{0}$ resolution function are taken from the fit of the mass spectrum for simulated events in the corresponding $Q^{2}$ interval. For the fits to the data, the value of the parameter $\sigma_{1}$ is modified to take into account the observed data-simulation difference in resolution: $\sigma_{1} \rightarrow$ $\sqrt{\sigma_{1}^{2}-(1.9 \mathrm{MeV})^{2}}$. The free parameters in the data fits are the number of signal events and two or three parameters, depending upon the description of the background shape. The numbers of signal events obtained from the fits using a linear background are listed in Table I. The difference between the fits for the two background hypotheses is used as an estimate of the systematic uncertainty associated with the unknown background shape. The two-photon

TABLE I. For each $Q^{2}$ interval, the number of events with $\pi^{0}$ obtained from the fit $\left(N_{\pi}\right)$, number of $e^{+} e^{-} \rightarrow e^{+} e^{-} \pi^{0} \pi^{0}$ background events $\left(N_{\mathrm{bkg}}\right)$, total efficiency correction $\left(\delta_{\text {total }}\right)$, number of signal events corrected for data/MC difference and resolution effects $\left(N_{\text {cor }}\right)$, and detection efficiency obtained from simulation $(\varepsilon)$. The quoted errors on $N_{\pi}$ and $N_{\text {cor }}$ are statistical and systematic,

\begin{tabular}{|c|c|c|c|c|c|}
\hline$Q^{2}$ interval $\left(\mathrm{GeV}^{2}\right)$ & $N_{\pi}$ & $N_{\mathrm{bkg}}$ & $\delta_{\text {total }}(\%)$ & $N_{\text {cor }}$ & $\varepsilon(\%)$ \\
\hline $4.0-4.5$ & $1645 \pm 45 \pm 4$ & $176 \pm 41$ & $-4.9 \pm 1.2$ & $1503 \pm 52 \pm 52$ & 5.2 \\
\hline $4.5-5.0$ & $1920 \pm 49 \pm 11$ & $254 \pm 54$ & $-5.5 \pm 1.1$ & $1740 \pm 58 \pm 70$ & 9.0 \\
\hline $5.0-5.5$ & $1646 \pm 46 \pm 5$ & $206 \pm 34$ & $-5.0 \pm 1.1$ & $1551 \pm 56 \pm 46$ & 10.3 \\
\hline $5.5-6.0$ & $1252 \pm 41 \pm 5$ & $175 \pm 30$ & $-5.5 \pm 1.0$ & $1139 \pm 50 \pm 40$ & 10.7 \\
\hline $6.0-7.0$ & $1891 \pm 50 \pm 2$ & $271 \pm 36$ & $-7.0 \pm 1.1$ & $1760 \pm 59 \pm 47$ & 11.5 \\
\hline $7.0-8.0$ & $1229 \pm 41 \pm 19$ & $150 \pm 29$ & $-7.5 \pm 1.0$ & $1160 \pm 50 \pm 44$ & 13.2 \\
\hline $8.0-9.0$ & $985 \pm 38 \pm 27$ & $125 \pm 24$ & $-7.3 \pm 0.9$ & $915 \pm 46 \pm 46$ & 15.3 \\
\hline $9.0-10.0$ & $829 \pm 34 \pm 8$ & $59 \pm 14$ & $-7.7 \pm 1.0$ & $849 \pm 43 \pm 23$ & 17.3 \\
\hline $10.0-11.0$ & $625 \pm 30 \pm 18$ & $47 \pm 13$ & $-8.3 \pm 1.1$ & $634 \pm 40 \pm 30$ & 18.6 \\
\hline $11.0-12.0$ & $448 \pm 26 \pm 3$ & $27 \pm 11$ & $-8.4 \pm 1.0$ & $484 \pm 35 \pm 16$ & 18.7 \\
\hline $12.0-13.5$ & $405 \pm 26 \pm 22$ & $51 \pm 12$ & $-8.1 \pm 0.9$ & $381 \pm 33 \pm 32$ & 17.2 \\
\hline $13.5-15.0$ & $289 \pm 22 \pm 14$ & $13 \pm 6$ & $-7.3 \pm 1.0$ & $304 \pm 28 \pm 20$ & 16.3 \\
\hline $15.0-17.0$ & $260 \pm 22 \pm 5$ & $14 \pm 6$ & $-6.7 \pm 1.0$ & $270 \pm 27 \pm 11$ & 15.4 \\
\hline $17.0-20.0$ & $235 \pm 21 \pm 2$ & $20 \pm 6$ & $-6.6 \pm 1.1$ & $234 \pm 25 \pm 10$ & 13.9 \\
\hline $20.0-25.0$ & $171 \pm 19 \pm 11$ & $5 \pm 4$ & $-6.6 \pm 1.3$ & $185 \pm 22 \pm 14$ & 11.4 \\
\hline $25.0-30.0$ & $36 \pm 12 \pm 2$ & $1 \pm 1$ & $-6.9 \pm 1.5$ & $36 \pm 14 \pm 3$ & 9.2 \\
\hline $30.0-40.0$ & $49 \pm 12 \pm 2$ & $2 \pm 6$ & $-6.3 \pm 1.8$ & $53 \pm 13 \pm 8$ & 7.3 \\
\hline
\end{tabular}
respectively. For $N_{\text {cor }}$ we quote only $Q^{2}$-dependent systematic errors. The $Q^{2}$-independent systematic error is $2.5 \%$. 

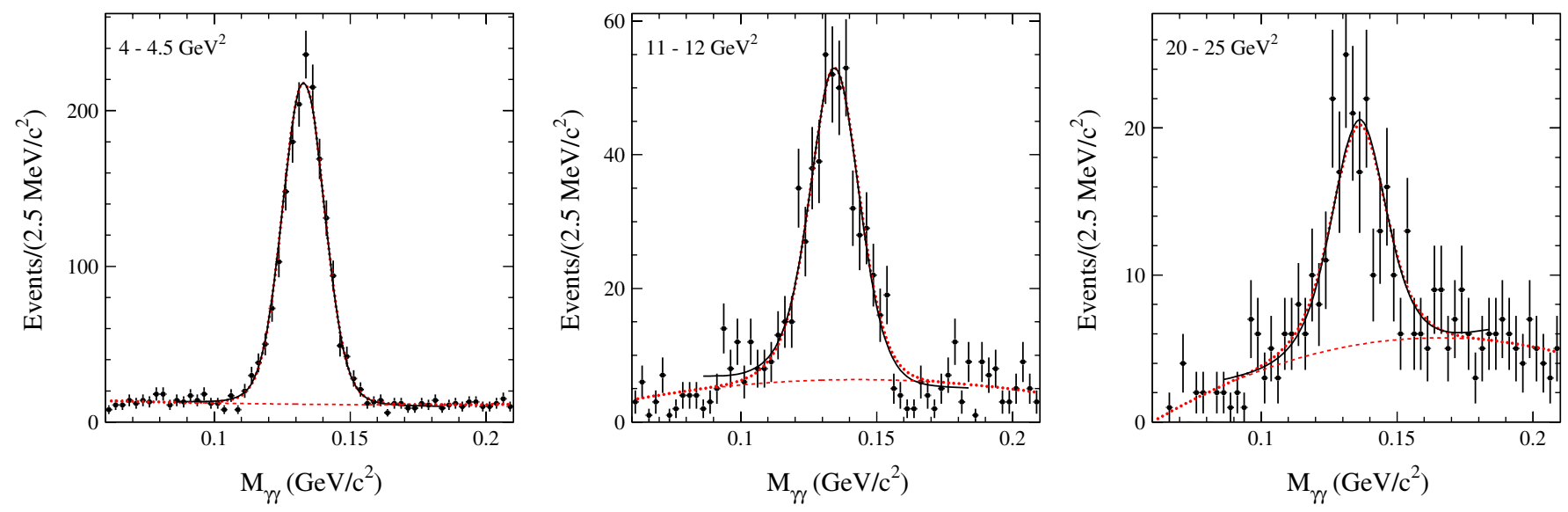

FIG. 6 (color online). The two-photon invariant mass spectra for data events from three representative $Q^{2}$ intervals. The solid (dotted) curve corresponds to the fit with a linear (quadratic) background shape. The dashed curve represents the fitted quadratic background.

mass spectra and fitted curves for three representative $Q^{2}$ intervals are shown in Fig. 6.

\section{PEAKING BACKGROUND ESTIMATION AND SUBTRACTION}

A background containing true $\pi^{0}$ 's might arise from processes such as beam-gas interaction, $e^{+} e^{-}$annihilation, and two-photon processes yielding higher multiplicity final states.

For beam-gas interactions, the total energy of the detected electron and $\pi^{0}$ should be less than the beam energy. In the energy spectrum of the $e \pi^{0}$ system, we do not see events with energy less than the beam energy. Therefore we conclude that the beam-gas background does not survive the selection criteria.

For events due to the signal process with tagged positron (electron), the momentum of the detected $e \pi^{0}$ system in the $e^{+} e^{-}$c.m. frame has a negative (positive) $z$ component, while events resulting from $e^{+} e^{-}$annihilation should be produced symmetrically. Events with the wrong sign of the $e \pi^{0}$ momentum $z$ component can therefore be used to estimate the background contribution from $e^{+} e^{-}$annihilation. The two-photon mass spectrum for such background events is shown in Fig. 7. The total number of wrong-sign events is about $3 \%$ of the selected signal event candidates. The spectrum is fitted using a sum of signal and background distributions as described in Sec. IV. The fit yields $6 \pm 16 \pi^{0}$ events. Assuming that the numbers of background events from $e^{+} e^{-}$annihilation in the wrong and right-sign data samples are approximately the same, we conclude that this background does not exceed $0.2 \%$ of signal events, and so is negligible. Nevertheless we have analyzed simulated events for the processes $e^{+} e^{-} \rightarrow B \bar{B}$, $e^{+} e^{-} \rightarrow q \bar{q}$, and $e^{+} e^{-} \rightarrow \tau^{+} \tau^{-}$. The number of simulated events for each reaction is close to the number of such events produced in the experiment. Seven events with a right-sign $z$ component of the $e \pi^{0}$ momentum (four from $\tau^{+} \tau^{-}$and three from $q \bar{q}$ ) satisfy the analysis selection criteria, while the number of accepted wrong-sign events is four. This supports the conclusion that the $e^{+} e^{-}$annihilation background is negligible.

The major source of peaking background, of order 10\%, is two-photon production of two $\pi^{0}$ 's. This background is clearly seen as a $\pi^{0}$ peak in the two-photon invariant mass spectrum for data events with two extra photons. The following procedure is used to estimate the $2 \pi^{0}$ background. We select a clean sample of $2 \pi^{0}$ events with the special selection criteria (described below) and measure the $Q^{2}$ distribution for these events $\left(N_{2 \pi, i}\right)$. Then we tune the MC simulation of the $e^{+} e^{-} \rightarrow e^{+} e^{-} \pi^{0} \pi^{0}$ reaction to reproduce the $2 \pi^{0}$ mass and $\pi^{0}$ angular distributions observed in data. Using the MC simulation we calculate the ratio $\left(\kappa_{i}\right)$ of the numbers of $2 \pi^{0}$ events selected with the standard and special criteria and estimate the number of

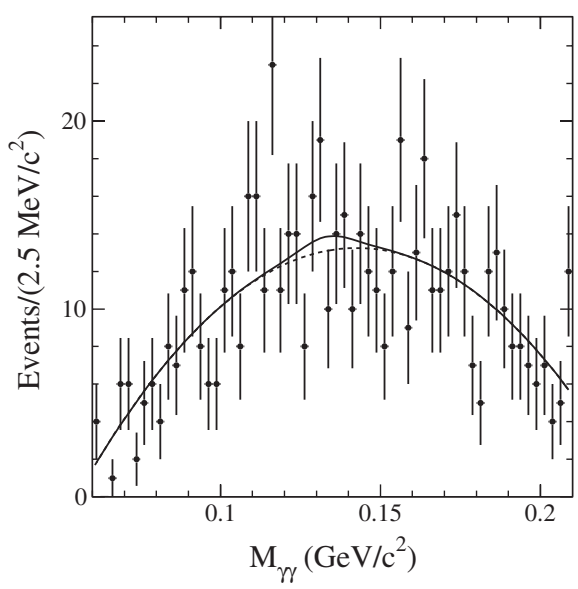

FIG. 7. The two-photon mass spectrum for data with the wrong sign of the $e \pi^{0}$ c.m. momentum component along the collision axis. The solid curve is the result of the fit described in the text. The dashed curve represents the fitted nonpeaking background. 


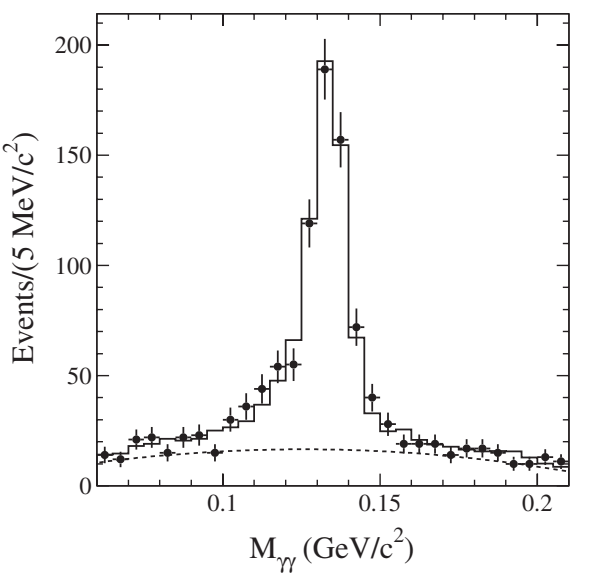

FIG. 8. The invariant mass spectrum of the extra $\pi^{0}$ candidate in $2 \pi^{0}$ data events (points with error bars). The histogram is the result of the fit using a sum of signal and background distributions. The dashed curve represents the fitted background distribution.

$2 \pi^{0}$ events for each $Q^{2}$ interval that satisfy the standard selection criteria as $\kappa_{i} N_{2 \pi, i}$.

To select $2 \pi^{0}$ events we remove the criteria on $r$ and $\cos \theta_{e \pi}^{*}$ and search for events with an extra $\pi^{0}$. The combinatorial background due to soft false photons is reduced by requiring that, in the laboratory, the energy of the extra $\pi^{0}$ be greater than $0.2 \mathrm{GeV}$, and that the energies of the decay photons be greater than $50 \mathrm{MeV}$. The mass of the first $\pi^{0}$ must be in the range $0.10-0.17 \mathrm{GeV} / c^{2}$. We calculate the parameters $\cos \theta^{*}$ and $r$ for the found $e \pi^{0} \pi^{0}$ system, and require $\left|\cos \theta_{e \pi \pi}^{*}\right|>0.99$ and $-0.025<r<$ 0.05 . The two-photon invariant mass spectrum for the extra $\pi^{0}$ candidates is shown in Fig. 8. The mass spectrum is fitted using a sum of signal and background distributions. The signal distribution is obtained from MC simulation for the $e^{+} e^{-} \rightarrow e^{+} e^{-} \pi^{0} \pi^{0}$ process. The background is described by a second order polynomial. This fitting procedure is performed for all $Q^{2}$ intervals. To estimate the systematic uncertainty due to the unknown background shape, we make two fits, one with a linear and one with a quadratic background. The difference in $\pi^{0}$ signal size between the fits is taken as a measure of systematic uncertainty.

The $2 \pi^{0}$ mass spectrum for selected $2 \pi^{0}$ events after background subtraction is shown in Fig. 9. The observed spectrum differs strongly from the spectrum measured in the no-tag mode [21], where the dominant mechanism of $2 \pi^{0}$ production is $\gamma \gamma \rightarrow f_{2}(1270) \rightarrow \pi^{0} \pi^{0}$. In the no-tag mode the $f_{2}(1270)$ meson is produced predominantly in the helicity-2 state with angular distribution $\sim \sin ^{4} \theta_{\pi \pi}$, where the $\theta_{\pi \pi}$ is the angle between the $\pi^{0}$ direction and the assumed $\gamma \gamma$ collision axis in the dipion rest frame. The $\cos \theta_{\pi \pi}$ distribution for selected $2 \pi^{0}$ events after background subtraction is shown in Fig. 10. It is seen that our criteria select events with $\theta_{\pi \pi}$ near zero and strongly

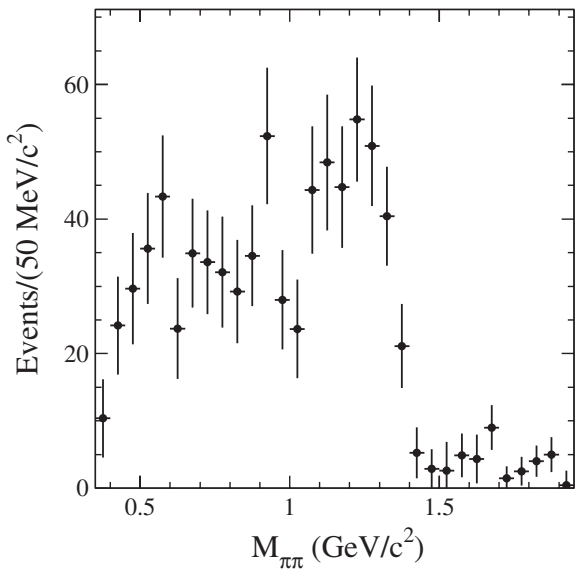

FIG. 9. The $2 \pi^{0}$ invariant mass spectrum for data.

suppress $f_{2}(1270)$ production in the helicity- 2 state. The spectrum in Fig. 9 contains three components: tensor $f_{2}(1270)$, scalar $f_{0}(980)$, and a broad bump below $0.8 \mathrm{GeV} / c^{2}$. We reweight the simulated events to reproduce the mass spectrum observed in data. Since the mass spectrum may change with $Q^{2}$, the reweighting is performed for two $Q^{2}$ intervals $\left(4<Q^{2}<10 \mathrm{GeV}^{2}\right.$ and $10<$ $Q^{2}<40 \mathrm{GeV}^{2}$ ) separately.

The simulated events are generated with isotropic $\pi^{0}$ angular distribution in the $2 \pi^{0}$ rest frame. Comparison of the simulated $\cos \theta_{\pi \pi}$ distribution with the data distribution is shown in Fig. 10. We reweight the $f_{2}(1270)$ subsample of simulated events so that the total MC simulated distribution of Fig. 10 matches the data. Using reweighted simulated events we calculate the $Q^{2}$ dependence of the scale factor $\kappa_{i}$ which varies from 2.4 at $Q^{2} \sim 5 \mathrm{GeV}^{2}$ to about 1 at $Q^{2}>15 \mathrm{GeV}^{2}$. The numbers of $2 \pi^{0}$ background events which satisfy our standard selection criteria are listed in Table I. The fraction of $2 \pi^{0}$ background events

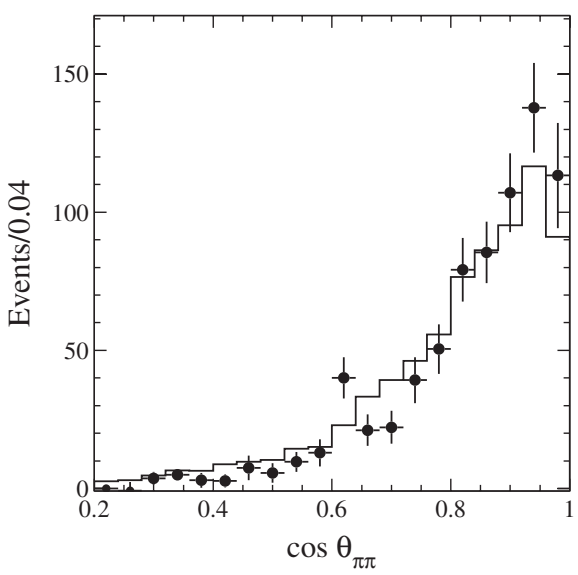

FIG. 10. The distribution of $\cos \theta_{\pi \pi}$ for data events (points with error bars) and simulated $e^{+} e^{-} \rightarrow e^{+} e^{-} \pi^{0} \pi^{0}$ events with isotropic $\pi^{0}$ angular distribution (histogram). 
in the $e \pi^{0}$ data sample changes from about $13 \%$ for $Q^{2}<$ $10 \mathrm{GeV}^{2}$ to $6 \%-7 \%$ for $Q^{2}>10 \mathrm{GeV}^{2}$.

A similar technique is used to search for background from the processes $e^{+} e^{-} \rightarrow e^{+} e^{-} \pi^{0} \eta, \eta \rightarrow \gamma \gamma$ and $e^{+} e^{-} \rightarrow e^{+} e^{-} \omega, \omega \rightarrow \pi^{0} \gamma$. We do not see a clear $\eta$ signal in the two-photon mass spectrum, nor do we see an $\omega$ signal in the $\pi^{0} \gamma$ mass spectrum; we estimate that these backgrounds do not exceed $5 \%$ of the $2 \pi^{0}$ background and thus are negligible.

\section{EFFICIENCY CORRECTION}

The values of the $\gamma^{*} \gamma \rightarrow \pi^{0}$ transition form factor in bins of $Q^{2}$ are determined from the ratio of the $Q^{2}$ distributions from data and MC simulation. The data distribution must be corrected to account for data-simulation difference in detector response:

$$
N_{i}^{\text {corr }}=\frac{N_{i}}{\prod_{j=1}^{4}\left(1+\delta_{j}^{i}\right)}
$$

where $i$ denotes the interval of $Q^{2}$ under consideration, and the $\delta_{j}^{i}$ 's are the corrections for the effects discussed in Secs. VI A, VIB, VIC, and VID.

\section{A. $\pi^{0}$ reconstruction efficiency}

A possible source of data-simulation difference is $\pi^{0}$ loss due to the merging of electromagnetic showers produced by the two photons from the $\pi^{0}$ decay, the loss of at least one of the decay photons, or the rejection of the $\pi^{0}$ because of the selection criteria. The $\pi^{0}$ efficiency is studied by using events produced in the ISR process $e^{+} e^{-} \rightarrow \omega \gamma$, where $\omega \rightarrow \pi^{+} \pi^{-} \pi^{0}$ [22]. These events can be selected and reconstructed using the measured parameters for only the two charged tracks and the ISR photon. Taking the ratio of the number of events with found $\pi^{0}$ to the total number of selected $e^{+} e^{-} \rightarrow \omega \gamma$ events, we measure the $\pi^{0}$ reconstruction efficiency. The events with reconstructed $\pi^{0}$ are selected with our standard criteria for the photons and the $\pi^{0}$, as described in Sec. III.

The ratio of the reconstruction efficiencies obtained in data and in simulation provides a $\pi^{0}$ efficiency correction. This correction, $\delta_{1}=\epsilon_{\text {data }} / \epsilon_{\mathrm{MC}}-1$, is shown as a function of $\pi^{0}$ laboratory energy in Fig. 11. The energy dependence is well described by a linear function. We estimate that the systematic uncertainty associated with this correction does not exceed $1 \%$.

To obtain the correction to the MC-estimated $\pi^{0}$ efficiency as a function of $Q^{2}$, we convolve the correction energy dependence of Fig. 11 with the $\pi^{0}$ energy spectrum in each $Q^{2}$ interval. The $Q^{2}$ dependence obtained is shown in Fig. 12. Only statistical errors are shown; the systematic error is estimated to be $1 \%$.

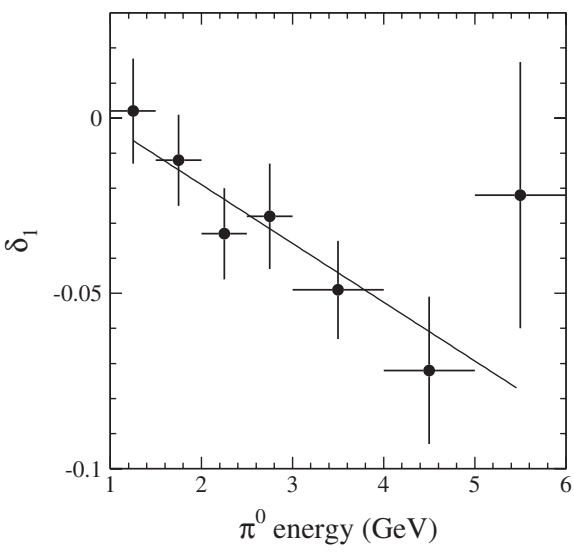

FIG. 11. The correction to the MC-estimated $\pi^{0}$ efficiency as a function of $\pi^{0}$ energy in the laboratory frame.

\section{B. Electron identification efficiency}

The average electron identification (EID) inefficiency in the signal MC simulation is about $1 \%$. To estimate the data-simulation difference in EID, we use VCS events which can be selected with negligible background without any EID requirements. The EID efficiency is determined as the ratio of the number of events with an identified electron to the total number of VCS events. The ratio of the efficiencies obtained from data and simulation gives the efficiency correction. We determine the correction as a function of the electron energy and polar angle and convolve this function with the electron energy and angular distributions for the process under study. The resulting $Q^{2}$ dependence of the efficiency correction is shown in Fig. 13.

\section{Trigger efficiency}

With the available statistics and the trigger configuration used, we cannot determine the trigger efficiency for the process under study by using the data. However, the trigger efficiency can be measured for the VCS process, which has a much larger cross section. The VCS events allow the

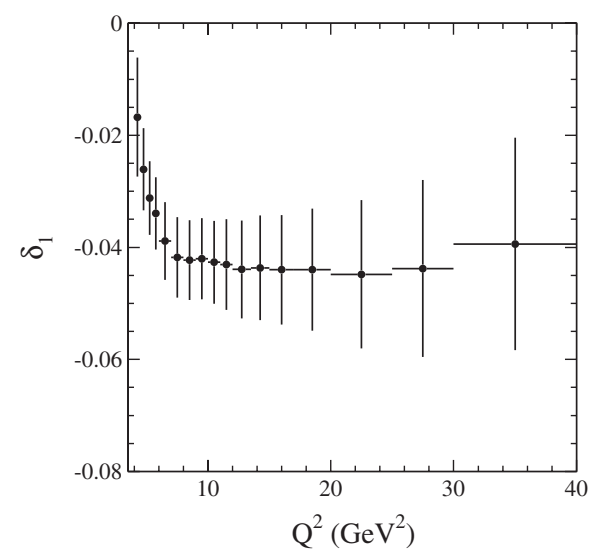

FIG. 12. The $Q^{2}$ dependence of the correction to the MCestimated efficiency for $\pi^{0}$. 


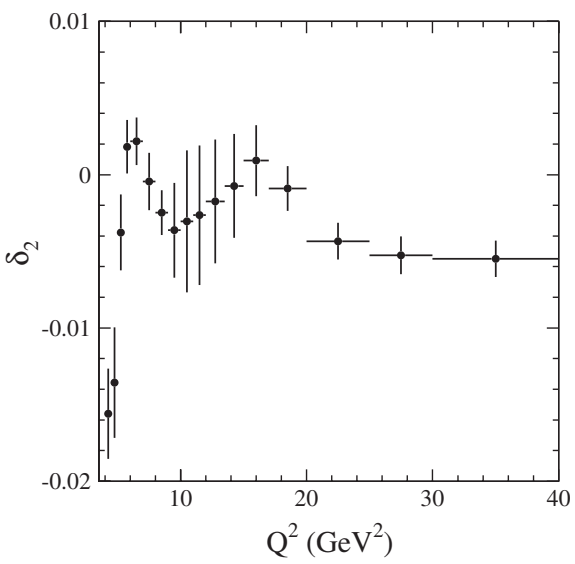

FIG. 13. The correction to the MC-estimated EID efficiency as a function of $Q^{2}$.

determination of the part of the trigger inefficiency related to the trigger track-finding algorithm. The remaining trigger inefficiency, which is related to the ability of the trigger cluster algorithm to separate nearby photons from $\pi^{0}$ decay, depends strongly on $\pi^{0}$ energy. Therefore the data-simulation difference can be estimated from comparison of the $\pi^{0}$ energy spectra in data and simulation.

The VCS events are selected with the criteria described in Sec. III after applying the $\pi^{0}$ requirements to the VCS photon. These events must satisfy a second trigger line that selects VCS events with an efficiency close to $100 \%$, but that is prescaled by a factor of 1000 . The trigger efficiency is determined as the fraction of selected events which pass the VirtualCompton filter. The ratio of the efficiencies obtained from data and simulation gives the efficiency correction. We find that the trigger efficiency depends strongly on electron scattering angle. When $\theta_{e}$ changes from 0.376 to $0.317 \mathrm{rad}$, the efficiency falls from $70 \%$ to $30 \%$ and the efficiency correction increases from $10 \%$ to $30 \%$. For this reason the events with $\theta_{e}<0.376 \mathrm{rad}$ were removed from the analysis data sample. Since the angular and energy distributions for the $\mathrm{VCS}$ and $e^{+} e^{-} \rightarrow e^{+} e^{-} \pi^{0}$ processes are different, we determine the correction as a function of $\cos \theta_{\gamma}$ and $\cos \theta_{e}$, separately for tagged electrons and positrons, and convolve this function with the $\cos \theta_{\pi}$ and $\cos \theta_{e}$ distributions for the process under study. The resulting $Q^{2}$ dependence of the efficiency correction is shown in Fig. 14. The corrections for events with a tagged electron or positron are also shown. The correction for tagged positron events is about $-2 \%$ and flat. For events with a tagged electron, the graph begins at $Q^{2}=7 \mathrm{GeV}^{2}$. The electron correction changes from $-8 \%$ at $Q^{2}=$ $7 \mathrm{GeV}^{2}$ to about $-1.5 \%$ at $Q^{2}=20 \mathrm{GeV}^{2}$ and higher.

The trigger inefficiency determined directly from $e^{+} e^{-} \rightarrow e^{+} e^{-} \pi^{0}$ simulation is compared to that calculated using simulated VCS events in Fig. 15. The discrepancy between the inefficiencies is $3 \%-4 \%$ for $\pi^{0}$ energies higher than $3 \mathrm{GeV}$, but increases to $30 \%$ for $E_{\pi}<2 \mathrm{GeV}$.

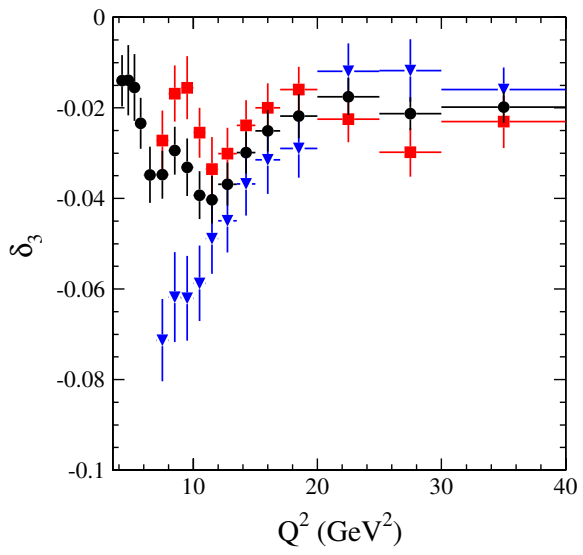

FIG. 14 (color online). The $Q^{2}$ dependence of the correction for trigger efficiency for events with a tagged electron (triangles) or positron (squares), and for all events (circles).

Figure 16 shows the $Q^{2}$ spectra for simulated signal pions of different energies. For each $Q^{2}$ interval the energy spectra for data and simulation should be identical. Any difference provides a measure of the quality of the trigger efficiency simulation. Using the fitting procedure described in Sec. IV, we determine the numbers of signal events in ten $Q^{2}$ intervals for six $\pi^{0}$ energy ranges (53 measurements, excluding cells with no events). We subtract the $2 \pi^{0}$ background and normalize the energy spectrum in each $Q^{2}$ interval so that its integral is unity. The same procedure is applied to simulated spectra after introducing the efficiency corrections for $\pi^{0}$ loss, EID and trigger inefficiency. The comparison of the normalized data and simulated spectra gives $\chi^{2} / \mathrm{ndf}=42.4 / 43$ (ndf $=$ number of degrees of freedom).

The $\pi^{0}$ energy spectrum summed over the $Q^{2}$ intervals from 4 to $12 \mathrm{GeV}^{2}$ is shown in Fig. 17. The simulated spectrum is the sum of the spectra normalized to the number of data events in each $Q^{2}$ interval. The shaded

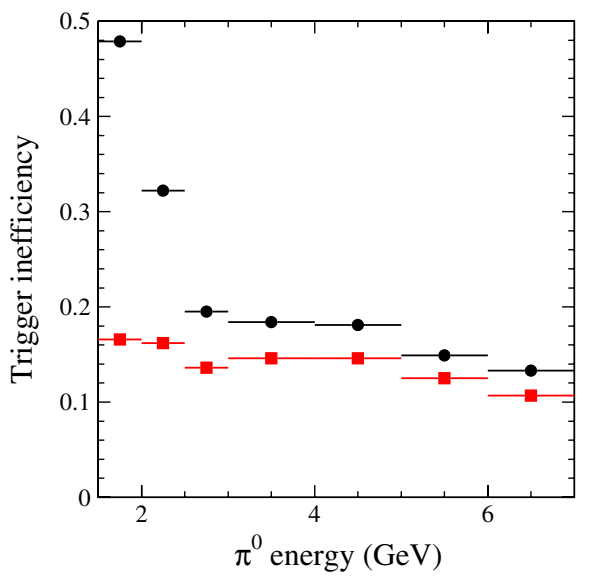

FIG. 15 (color online). The trigger inefficiency as a function of $\pi^{0}$ energy determined directly from $e^{+} e^{-} \rightarrow e^{+} e^{-} \pi^{0}$ simulation (circles) and from simulated VCS events (squares). 


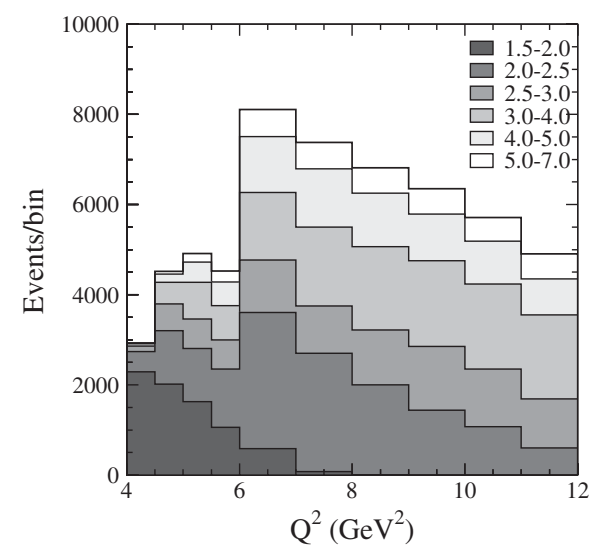

FIG. 16. The $Q^{2}$ spectrum for simulated signal events. The shading represents the contributions from the different $\pi^{0}$ energy ranges (in $\mathrm{GeV}$ ) indicated by the key.

boxes represent the uncertainties in the efficiency corrections. The ratio of the data and simulated spectra agrees with unity with $\chi^{2} / \mathrm{ndf}=5.9 / 5$. Since the spectra for data and simulation are in reasonable agreement, we conclude that the simulation reproduces the discrepancy in trigger inefficiency, and so there is no need to introduce an extra efficiency correction. However, we introduce an extra systematic uncertainty due to trigger inefficiency, which is conservatively estimated to be $2 \%$, i.e., half of the difference between the VCS and $e^{+} e^{-} \rightarrow e^{+} e^{-} \pi^{0}$ trigger inefficiencies for high energy $\pi^{0}$ 's (see Fig. 15).

Since the energy and angular distributions, and the trigger efficiency correction are very different for events with a tagged electron or a tagged positron, it is interesting to compare the $e^{+} e^{-} \rightarrow e^{+} e^{-} \pi^{0}$ differential cross sections measured for electron-tagged and positron-tagged events. To do this we subtract the $2 \pi^{0}$ background and apply the efficiency correction separately to events with tagged electron and positron. The ratio of the cross

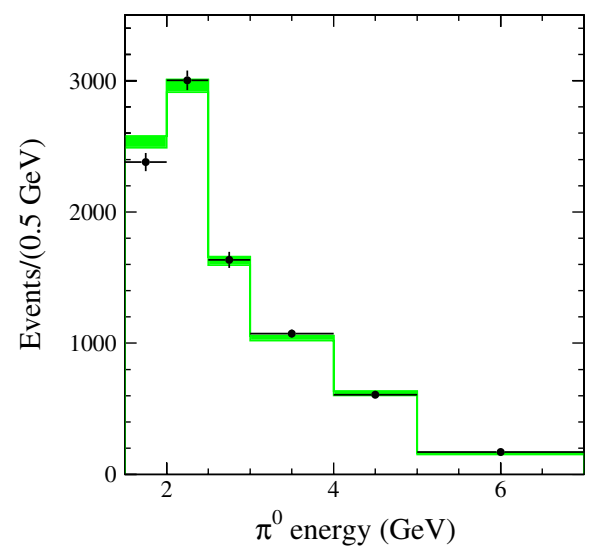

FIG. 17 (color online). The $\pi^{0}$ energy spectrum for data (points with error bars) and signal simulation (histogram). The shaded boxes represent the uncertainties associated with the efficiency correction procedure.

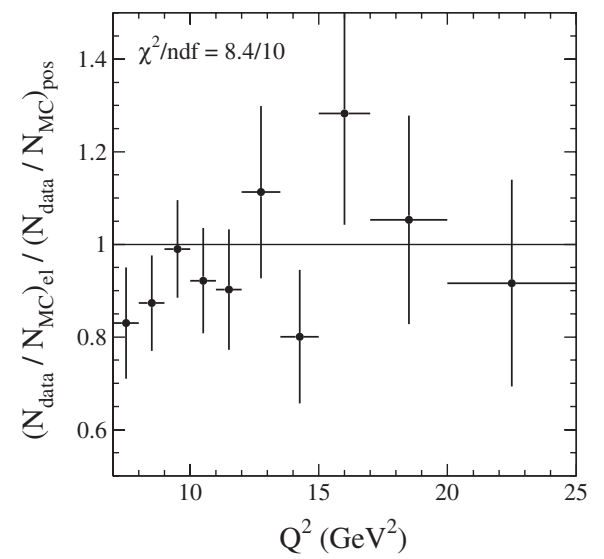

FIG. 18. The $Q^{2}$ dependence of the double ratio defined in the text to compare the cross section for selected signal events with a tagged electron to that for events with a tagged positron.

sections is then calculated as the double ratio $\left(N_{\text {data }} / N_{\text {MC }}\right)_{\text {electrons }} /\left(N_{\text {data }} / N_{\text {MC }}\right)_{\text {positrons }}$ (Fig. 18) and is found to be in reasonable agreement with unity.

\section{Requirements on $\boldsymbol{r}$ and $\cos \theta_{e \pi}^{*}$}

To estimate possible systematic uncertainty due to the requirement $-0.025<r<0.05$, we study events in the range $0.05<r<0.075$ [see Eq. (3) and Fig. 3]. Assuming that the efficiency corrections are the same for events from the two $r$ ranges, and subtracting $2 \pi^{0}$ background, we determine the double ratio $\left(N_{\text {data }} / N_{\text {MC }}\right)_{0.05<r<0.075} /\left(N_{\text {data }} / N_{\text {MC }}\right)_{-0.025<r<0.05}$ as a function of $Q^{2}$. The ratio is consistent with unity $\left(\chi^{2} / \mathrm{ndf}=9.3 / 15\right)$, so we conclude that the MC simulation reproduces the shape of the $r$ distribution.

We also study the effect of the $\left|\cos \theta_{e \pi}^{*}\right|>0.99$ criterion by changing the value to 0.98 and 0.95 . The ratio of the numbers of events with $0.98<\left|\cos \theta_{e \pi}^{*}\right|<0.99$ and $\left|\cos \theta_{e \pi}^{*}\right|>0.99$ is found to be $0.013 \pm 0.003$ in data and $0.0074 \pm 0.0004$ in simulation. The corresponding values for $0.95<\left|\cos \theta_{e \pi}^{*}\right|<0.99$ are $0.018 \pm 0.002$ and $0.0103 \pm 0.0005$. Since the observed data-simulation difference does not exceed 1\%, we do not introduce an efficiency correction, but consider this difference (1\%) as a measure of systematic uncertainty due to the $\cos \theta_{e \pi}^{*}$ criterion.

\section{E. Effect of the beam-induced background}

The quality of simulation of beam-generated spurious track and photons is checked using practically backgroundfree VCS events. The simulated distributions of the number of extra photons, their energies, number of extra tracks, and their momenta are found to be in a reasonable agreement with those from data.

The average values of the parameters for data and simulation are listed in Table II. We find that the values of these parameters are independent of $Q^{2}$. 
TABLE II. The average number of extra photons $\left(N_{\mathrm{ph}}\right)$, the average energy of extra photon $\left(E_{\mathrm{ph}}\right)$, the fraction of events with extra track $\left(f_{\text {trk }}\right)$, and the average momentum of extra track $p_{\text {trk }}$ in data and simulation for VCS events.

\begin{tabular}{lcccr}
\hline \hline & $N_{\mathrm{ph}}$ & $E_{\mathrm{ph}}, \mathrm{MeV}$ & $f_{\text {trk }}, \%$ & $p_{\text {trk }}, \mathrm{MeV}$ \\
\hline Data & $2.31 \pm 0.01$ & $40.2 \pm 0.1$ & $1.62 \pm 0.03$ & $196 \pm 4$ \\
MC & $2.27 \pm 0.01$ & $38.4 \pm 0.1$ & $1.81 \pm 0.04$ & $205 \pm 4$ \\
\hline \hline
\end{tabular}

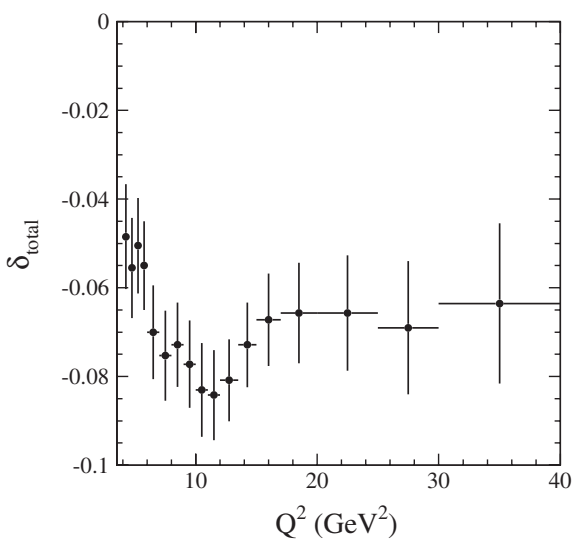

FIG. 19. The $Q^{2}$ dependence of the total efficiency correction.

The background conditions changed considerably over the course of the experiment. For example, the average number of extra photons varied from 1.5 for 2001 data to 3.5 for 2007 data. It was tested that simulation reproduces this variation well. It should be noted that our selection criteria are only slightly sensitive to beam-induced background. The relative difference between the detection efficiencies calculated with 2001 and 2007 background conditions is found to be $(-1.2 \pm 0.9) \%$. Since the simulation reproduces the beam-induced background and its variation, we do not introduce any systematic uncertainty associated with the effects of the beam-induced background.

The total efficiency correction as a function of $Q^{2}$ is shown in Fig. 19, where the error bars are of statistical origin. The systematic uncertainty, which is independent of $Q^{2}$, is $2.5 \%$ and takes into account the uncertainties in the determination of $\pi^{0}$ loss $(1 \%)$ and trigger inefficiency $(2 \%)$, and the uncertainty due to the $\left|\cos \theta_{e \pi}^{*}\right|>0.99$ requirement $(1 \%)$. The values of the total efficiency correction and their statistical errors are listed in Table I.

\section{CROSS SECTION AND FORM FACTOR}

The Born differential cross section for $e^{+} e^{-} \rightarrow e^{+} e^{-} \pi^{0}$ is calculated as

$$
\frac{d \sigma}{d Q^{2}}=\frac{N_{\mathrm{cor}} / \Delta Q^{2}}{\varepsilon R L}
$$

where $N_{\text {cor }}$ is the number of signal events corrected for data-simulation difference and resolution effects (Table I), $\Delta Q^{2}$ is the relevant $Q^{2}$ interval, $L$ is the total integrated luminosity $\left(442 \mathrm{fb}^{-1}\right), \varepsilon$ is the detection efficiency as a function of $Q^{2}$, and $R$ is a radiative correction factor accounting for distortion of the $Q^{2}$ spectrum due to the emission of photons from the initial state particles and for vacuum polarization effects. The detection efficiency is obtained from simulation. Its $Q^{2}$ dependence is shown in Fig. 4 and listed in Table I.

The radiative correction factor is determined using generator-only simulation. The $Q^{2}$ spectrum is generated using only the Born amplitude for the $e^{+} e^{-} \rightarrow e^{+} e^{-} \pi^{0}$ process, and then again using a model with radiative corrections included. The $Q^{2}$ dependence of the radiative correction factor, evaluated as the ratio of the second spectrum to the first, is shown in Fig. 20. The $Q^{2}$ dependence is fitted by the function $a /\left(1+b Q^{\gamma}\right)$. The accuracy of the radiative correction calculation is estimated to be $1 \%$ [15]. Note that the value of $R$ depends on the requirement on the extra photon energy. The $Q^{2}$ dependence obtained corresponds to the criterion $r=2 E_{\gamma}^{*} / \sqrt{s}<0.1$ imposed in the simulation.

The corrected mass spectrum $\left(N_{\text {cor }}\right)$ is obtained from the measured spectrum $\left(N_{\text {rec }}\right)$ by dividing by the efficiency correction factor [see Eq. (5)] and unfolding the effect of $Q^{2}$ resolution. Using MC simulation, a migration matrix $A$ is obtained, which represents the probability that an event with true $Q^{2}$ in interval $j$ is reconstructed in interval $i$ :

$$
N_{\mathrm{rec}, i}=\sum_{j} A_{i j} N_{\mathrm{cor}, j}
$$

In the case of extra photon emission, $Q_{\text {true }}^{2}$ is calculated as $-\left(p-p^{\prime}-k\right)^{2}$, where $k$ is the photon four-momentum; $\varepsilon$ and $R$ in Eq. (6) are functions of $Q_{\text {true }}^{2}$. The $Q^{2}$ resolution varies from about $0.05 \mathrm{GeV}^{2}$ at $Q^{2}=5 \mathrm{GeV}^{2}$ to $0.25 \mathrm{GeV}^{2}$ at $Q^{2}=25 \mathrm{GeV}^{2}$. As the chosen $Q^{2}$ interval size significantly exceeds the resolution for all $Q^{2}$, the migration matrix is nearly diagonal, with diagonal values

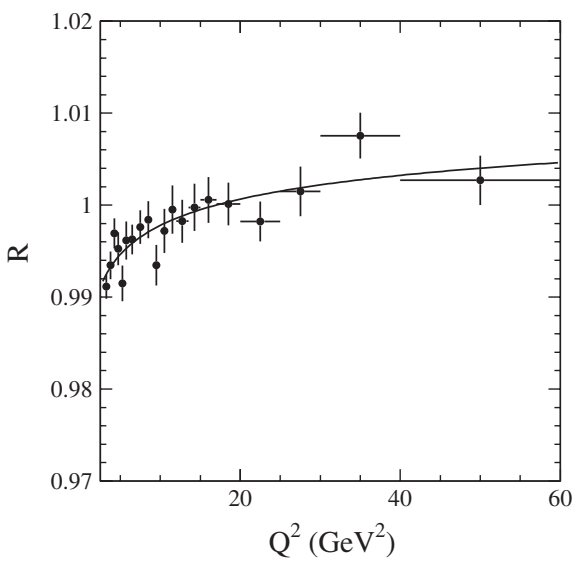

FIG. 20. The $Q^{2}$ dependence of the radiative correction factor. 
$\sim 0.9$, and next-to-diagonal values $\sim 0.05$. We unfold the $Q^{2}$ spectrum by applying the inverse of the migration matrix to the measured spectrum. The procedure changes the shape of the $Q^{2}$ distribution insignificantly, but increases the errors (by about 20\%) and their correlations. The corrected $Q^{2}$ spectrum is listed in Table I.

The values of the differential cross section are listed in Table III. The quoted errors are statistical and systematic. The latter includes only $Q^{2}$-dependent errors: the systematic uncertainty in the number of signal events and the statistical error in the detection efficiency determined from MC simulation. The $Q^{2}$-independent systematic error is equal to $3 \%$ and includes the systematic uncertainties in the efficiency correction $(2.5 \%)$ and in the radiative correction factor $(1 \%)$, and the uncertainty in the integrated luminosity (1\%).

The measured differential cross section at the Born level is shown in Fig. 21, together with CLEO data [12] for $Q^{2}>$ $4 \mathrm{GeV}^{2}$.

Because of the strong nonlinear dependence of the cross section on $Q^{2}$, the effective value of $Q^{2}$ corresponding to the measured cross section differs from the center of the $Q^{2}$ interval. We parametrize the measured cross section by a smooth function, reweight the $Q^{2}$ distribution in simulation to be consistent with data, and calculate the weighted average $\left(\bar{Q}^{2}\right)$ for each mass interval. The values of $\bar{Q}^{2}$ are listed in Table III.

TABLE III. The $Q^{2}$ interval, the weighted average $Q^{2}$ value for the interval $\left(\bar{Q}^{2}\right)$, the $e^{+} e^{-} \rightarrow e^{+} e^{-} \pi^{0}$ cross section $\left(d \sigma / d Q^{2}\left(\bar{Q}^{2}\right)\right)$, and the product of the $\gamma \gamma^{*} \rightarrow \pi^{0}$ transition form factor $F\left(\bar{Q}^{2}\right)$ and $\bar{Q}^{2}$. The quoted errors are statistical and systematic for the cross section, and combined for the form factor. In the table we quote only $Q^{2}$-dependent systematic errors. The $Q^{2}$-independent systematic error is $3 \%$ for the cross section and $2.3 \%$ for the form factor.

\begin{tabular}{lccc}
\hline \hline $\begin{array}{l}Q^{2} \text { interval } \\
\left(\mathrm{GeV}^{2}\right)\end{array}$ & $\begin{array}{c}\bar{Q}^{2} \\
\left(\mathrm{GeV}^{2}\right)\end{array}$ & $\begin{array}{c}d \sigma / d Q^{2}\left(\bar{Q}^{2}\right) \\
\left(\mathrm{fb} / \mathrm{GeV}^{2}\right)\end{array}$ & $\begin{array}{c}\bar{Q}^{2}\left|F\left(\bar{Q}^{2}\right)\right| \\
(\mathrm{MeV})\end{array}$ \\
\hline $4.0-4.5$ & 4.24 & $131.4 \pm 4.6 \pm 5.0$ & $150.4 \pm 3.9$ \\
$4.5-5.0$ & 4.74 & $87.7 \pm 2.9 \pm 3.7$ & $149.1 \pm 4.1$ \\
$5.0-5.5$ & 5.24 & $68.4 \pm 2.5 \pm 2.2$ & $157.4 \pm 3.9$ \\
$5.5-6.0$ & 5.74 & $48.3 \pm 2.1 \pm 1.8$ & $156.0 \pm 4.5$ \\
$6.0-7.0$ & 6.47 & $34.8 \pm 1.2 \pm 1.0$ & $163.5 \pm 3.6$ \\
$7.0-8.0$ & 7.47 & $20.01 \pm 0.86 \pm 0.79$ & $160.6 \pm 4.7$ \\
$8.0-9.0$ & 8.48 & $13.60 \pm 0.69 \pm 0.70$ & $167.3 \pm 6.0$ \\
$9.0-10.0$ & 9.48 & $11.11 \pm 0.56 \pm 0.32$ & $185.3 \pm 5.5$ \\
$10.0-11.0$ & 10.48 & $7.73 \pm 0.48 \pm 0.38$ & $186.6 \pm 7.6$ \\
$11.0-12.0$ & 11.49 & $5.86 \pm 0.42 \pm 0.21$ & $191.6 \pm 7.8$ \\
$12.0-13.5$ & 12.71 & $3.35 \pm 0.29 \pm 0.28$ & $175.0 \pm 11.0$ \\
$13.5-15.0$ & 14.22 & $2.82 \pm 0.26 \pm 0.19$ & $198.0 \pm 12.0$ \\
$15.0-17.0$ & 15.95 & $1.99 \pm 0.20 \pm 0.09$ & $208.0 \pm 12.0$ \\
$17.0-20.0$ & 18.40 & $1.27 \pm 0.14 \pm 0.06$ & $220.0 \pm 13.0$ \\
$20.0-25.0$ & 22.28 & $0.73 \pm 0.09 \pm 0.06$ & $245.0 \pm 18.0$ \\
$25.0-30.0$ & 27.31 & $0.18 \pm 0.07 \pm 0.02$ & $181.0_{-40}^{+33.0}$ \\
$30.0-40.0$ & 34.36 & $0.16 \pm 0.04 \pm 0.02$ & $285.0_{-45.0}^{+39.0}$ \\
\hline \hline
\end{tabular}

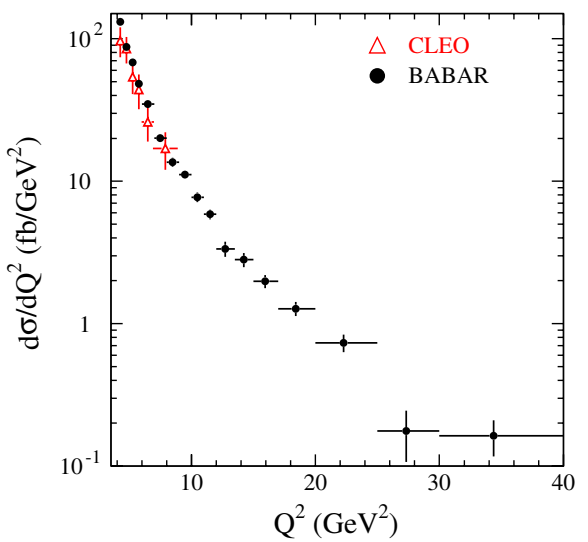

FIG. 21 (color online). The $e^{+} e^{-} \rightarrow e^{+} e^{-} \pi^{0}$ differential cross section obtained in this experiment compared to that from the CLEO experiment [12].

Since the requirement on $\cos \theta_{e \pi}^{*}$ limits the momentum transfer to the untagged electron, we measure the cross section for the restricted $q_{2}^{2}$ range $\left|q_{2}^{2}\right|<a_{\max }$. The value of $a_{\max }$ is determined from the $q_{2}^{2}$ dependence of the detection efficiency $\left[\varepsilon\left(a_{\max }\right)=50 \%\right]$ and is equal to $0.18 \mathrm{GeV}^{2}$.

To extract the transition form factor we compare the measured and the calculated values of the cross section. The simulation uses a constant form factor $F_{\mathrm{MC}}$. Therefore the measured form factor is determined from

$$
F^{2}\left(Q^{2}\right)=\frac{\left(d \sigma / d Q^{2}\right)_{\mathrm{data}}}{\left(d \sigma / d Q^{2}\right)_{\mathrm{MC}}} F_{\mathrm{MC}}^{2}
$$

The calculated cross section $\left(d \sigma / d Q^{2}\right)_{\mathrm{MC}}$ has a modeldependent uncertainty due to the unknown dependence on the momentum transfer to the untagged electron. We use a $q_{2}^{2}$-independent form factor, which corresponds to the QCD-inspired model $F\left(q_{1}^{2}, q_{2}^{2}\right) \propto 1 /\left(q_{1}^{2}+q_{2}^{2}\right) \approx 1 / q_{1}^{2}$ [23]. Using the vector dominance model with the form factor $F\left(q_{2}^{2}\right) \propto 1 /\left(1-q_{2}^{2} / m_{\rho}^{2}\right)$, where $m_{\rho}$ is $\rho$ meson mass, leads to a decrease of the cross section by $3.5 \%$. This difference is considered to be an estimate of model uncertainty due to the unknown $q_{2}^{2}$ dependence. However, it should be noted that this estimate depends strongly on the limit on $q_{2}^{2}$. The value of $3.5 \%$ is obtained with $\left|q_{2}^{2}\right|<$ $0.18 \mathrm{GeV}^{2}$. For a less stringent $q_{2}^{2}$ constraint, for example $\left|q_{2}^{2}\right|<0.6 \mathrm{GeV}^{2}$, the difference between the calculated cross sections reaches $7.5 \%$.

The values of the form factor obtained, represented in the form $\bar{Q}^{2}\left|F\left(\bar{Q}^{2}\right)\right|$, are listed in Table III and shown in Fig. 22. For the form factor we quote the combined error, for which the statistical and $Q^{2}$-dependent systematic uncertainties are added in quadrature. The $Q^{2}$-independent systematic error is $2.3 \%$, and includes the uncertainty on the measured differential cross section, and the modeldependent uncertainty due to the unknown $q_{2}^{2}$ dependence. 


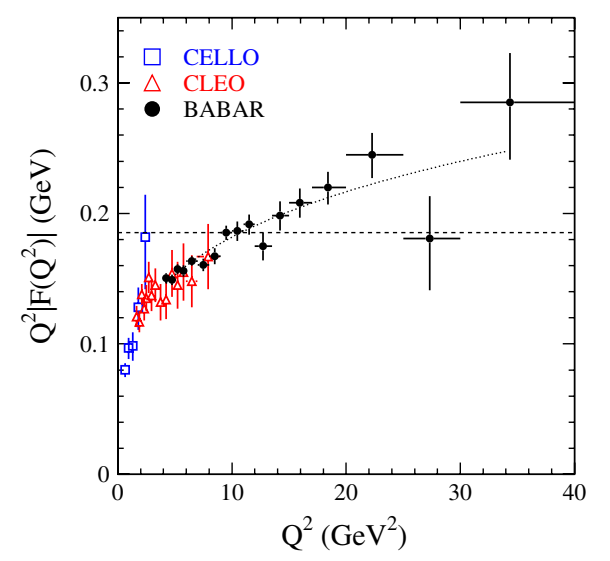

FIG. 22 (color online). The $\gamma \gamma^{*} \rightarrow \pi^{0}$ transition form factor multiplied by $Q^{2}$. The dashed line indicates the asymptotic limit for the form factor. The dotted curve shows the interpolation given by Eq. (9).

\section{CONCLUSIONS}

We have studied the $e^{+} e^{-} \rightarrow e^{+} e^{-} \pi^{0}$ reaction in the single tag mode and measured the differential cross section $\left(d \sigma / d Q^{2}\right)$ and the $\gamma \gamma^{*} \rightarrow \pi^{0}$ transition form factor $F\left(Q^{2}\right)$ for the momentum transfer range from 4 to $40 \mathrm{GeV}^{2}$. For the latter, the comparison of our results with previous measurements $[11,12]$ is shown in Fig. 22. In the $Q^{2}$ range from 4 to $9 \mathrm{GeV}^{2}$ our results are in reasonable agreement with the measurements by the CLEO collaboration [12], but have significantly better precision. We also significantly extend the $Q^{2}$ region over which the form factor is measured.

To effectively describe the $Q^{2}$ dependence of the form factor in the range $4-40 \mathrm{GeV}^{2}$, we fit the function

$$
Q^{2}\left|F\left(Q^{2}\right)\right|=A\left(\frac{Q^{2}}{10 \mathrm{GeV}^{2}}\right)^{\beta}
$$

to our data. The values obtained for the parameters are $A=$ $0.182 \pm 0.002 \mathrm{GeV}$, and $\beta=0.25 \pm 0.02$. The fit result is shown in Fig. 22 by the dotted curve. The effective $Q^{2}$ dependence of the form factor $\left(\sim 1 / Q^{3 / 2}\right)$ differs significantly from the leading order $\mathrm{pQCD}$ prediction $\left(\sim 1 / Q^{2}\right)$ [see Eq. (2)], demonstrating the importance of higher-order pQCD and power corrections in the $Q^{2}$ region under study.

The horizontal dashed line in Fig. 22 indicates the asymptotic limit $Q^{2} F\left(Q^{2}\right)=\sqrt{2} f_{\pi} \approx 0.185 \mathrm{GeV}$ for $Q^{2} \rightarrow \infty$, predicted by $\mathrm{pQCD}$ [2]. The measured form factor exceeds the limit for $Q^{2}>10 \mathrm{GeV}^{2}$. This contradicts most models for the pion distribution amplitude (see, e.g., Ref. [24] and references therein), which give form factors approaching the asymptotic limit from below.

The comparison of the form-factor data to the predictions of some theoretical models is shown in Fig. 23. The calculation of [8] was performed by Bakulev, Mikhailov, and Stefanis using the light-cone sum rule method [4,25] at next-to-leading order (NLO) pQCD; the power correction due to the twist- 4 contribution [25] was also taken into

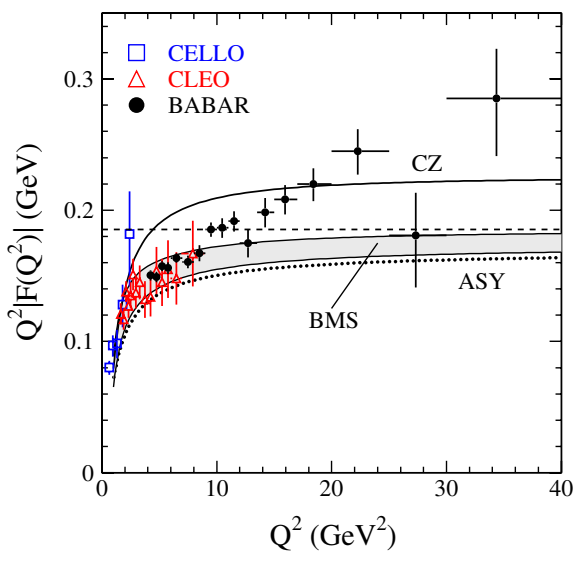

FIG. 23 (color online). The $\gamma \gamma^{*} \rightarrow \pi^{0}$ transition form factor multiplied by $Q^{2}$. The dashed line indicates the asymptotic limit for the form factor. The solid and dotted lines show the predictions for the form factor [8] for the CZ [26] and asymptotic (ASY) [27] models for the pion distribution amplitude, respectively. The shaded band represents the prediction for the BMS [28] pion DA model.

account. Their results are shown for the ChernyakZhitnitsky DA (CZ) [26], the asymptotic DA (ASY) [27], and the DA derived from QCD sum rules with nonlocal condensates (BMS) [28].

For all three DAs the $Q^{2}$ dependence is almost flat for $Q^{2} \geq 10 \mathrm{GeV}^{2}$, whereas the data show significant growth of the form factor between 8 and $20 \mathrm{GeV}^{2}$. This indicates that the NLO pQCD approximation with twist-4 power correction, which has been widely used for the description of the form-factor measurements by the CLEO collaboration [12], is inadequate for $Q^{2}$ less than $\sim 15 \mathrm{GeV}^{2}$. In the $Q^{2}$ range from 20 to $40 \mathrm{GeV}^{2}$, uncertainties due to higherorder pQCD and power corrections are expected to be relatively small. Here, our data lie above the asymptotic limit, as does the prediction of the $\mathrm{CZ}$ model.

\section{ACKNOWLEDGMENTS}

We thank V.L. Chernyak for useful discussions, and A. P. Bakulev, S. V. Mikhailov, and N. G. Stefanis for providing us the NLO pQCD calculation of the transition form factor. We are grateful for the extraordinary contributions of our PEP-II colleagues in achieving the excellent luminosity and machine conditions that have made this work possible. The success of this project also relies critically on the expertise and dedication of the computing organizations that support BABAR. The collaborating institutions wish to thank SLAC for its support and the kind hospitality extended to them. This work is supported by the U.S. Department of Energy and National Science Foundation, the Natural Sciences and Engineering Research Council (Canada), the Commissariat à l'Energie Atomique and Institut National de Physique Nucléaire et de Physique des Particules (France), the Bundesministerium für 
Bildung und Forschung and Deutsche Forschungsgemeinschaft (Germany), the Istituto Nazionale di Fisica Nucleare (Italy), the Foundation for Fundamental Research on Matter (The Netherlands), the Research Council of Norway, the Ministry of Science and Technology of the
Russian Federation, Ministerio de Educación y Ciencia (Spain), and the Science and Technology Facilities Council (United Kingdom). Individuals have received support from the Marie-Curie IEF program (European Union) and the A. P. Sloan Foundation.
[1] S. J. Brodsky, T. Kinoshita, and H. Terazawa, Phys. Rev. D 4, 1532 (1971).

[2] G. P. Lepage and S. J. Brodsky, Phys. Rev. D 22, 2157 (1980).

[3] P. Kroll and M. Raulfs, Phys. Lett. B 387, 848 (1996).

[4] A. Schmedding and O. I. Yakovlev, Phys. Rev. D 62, 116002 (2000).

[5] N. G. Stefanis, W. Schroers, and H. C. Kim, Eur. Phys. J. C 18, 137 (2000).

[6] E. Ruiz Arriola and W. Broniowski, Phys. Rev. D 66, 094016 (2002).

[7] A. P. Bakulev and S. V. Mikhailov, Phys. Rev. D 65, 114511 (2002).

[8] A. P. Bakulev, S. V. Mikhailov, and N. G. Stefanis, Phys. Rev. D 67, 074012 (2003); Phys. Lett. B 578, 91 (2004).

[9] S. S. Agaev, Phys. Rev. D 72, 114010 (2005); 73, 059902 (E) (2006).

[10] F. Feng, J. P. Ma, and Q. Wang, J. High Energy Phys. 06 (2007) 039.

[11] H. J. Behrend et al. (CELLO Collaboration), Z. Phys. C 49, 401 (1991).

[12] J. Gronberg et al. (CLEO Collaboration), Phys. Rev. D 57, 33 (1998).

[13] B. Aubert et al. (BABAR Collaboration), Nucl. Instrum. Methods Phys. Res., Sect. A 479, 1 (2002).

[14] V. M. Budnev, I. F. Ginzburg, G. V. Meledin, and V. G. Serbo, Phys. Rep. 15, 181 (1975).
[15] S. Ong and P. Kessler, Phys. Rev. D 38, 2280 (1988).

[16] S. Agostinelli et al. (GEANT4 Collaboration), Nucl. Instrum. Methods Phys. Res., Sect. A 506, 250 (2003).

[17] T. Sjostrand, Comput. Phys. Commun. 82, 74 (1994).

[18] S. Jadach, B. F. L. Ward, and Z. Was, Comput. Phys. Commun. 130, 260 (2000).

[19] D. J. Lange, Nucl. Instrum. Methods Phys. Res., Sect. A 462, 152 (2001).

[20] A.D. Bukin, Report No. INP-2007-035, Novosibirsk, 2007.

[21] H. Marsiske et al. (Crystal Ball Collaboration), Phys. Rev. D 41, 3324 (1990); S. Uehara et al. (Belle Collaboration), Phys. Rev. D 78, 052004 (2008).

[22] B. Aubert et al. (BABAR Collaboration), Phys. Rev. D 70, 072004 (2004).

[23] G. Kopp, T. F. Walsh, and P. M. Zerwas, Nucl. Phys. B70, 461 (1974).

[24] N. G. Stefanis, Nucl. Phys. Proc. Suppl. 181-182, 199 (2008).

[25] A. Khodjamirian, Eur. Phys. J. C 6, 477 (1999).

[26] V. L. Chernyak and A. R. Zhitnitsky, Nucl. Phys. B201, 492 (1982); B214, 547(E) (1983).

[27] G. P. Lepage and S. J. Brodsky, Phys. Lett. 87B, 359 (1979).

[28] A. P. Bakulev, S. V. Mikhailov, and N. G. Stefanis, Phys. Lett. B 508, 279 (2001); 590, 309(E) (2004). 